\title{
Tagged ozone mechanism for MOZART-4, CAM-chem and other chemical transport models
}

\author{
L. K. Emmons ${ }^{1}$, P. G. Hess ${ }^{2}$, J.-F. Lamarque ${ }^{1}$, and G. G. Pfister ${ }^{1}$ \\ ${ }^{1}$ Atmospheric Chemistry Division, National Center for Atmospheric Research, Boulder, CO, USA \\ ${ }^{2}$ Department of Biological and Environmental Engineering, Cornell University, Ithaca, NY, USA
}

Correspondence to: L. K. Emmons (emmons@ucar.edu)

Received: 5 July 2012 - Published in Geosci. Model Dev. Discuss.: 24 July 2012

Revised: 31 October 2012 - Accepted: 2 November 2012 - Published: 6 December 2012

\begin{abstract}
A procedure for tagging ozone produced from NO sources through updates to an existing chemical mechanism is described, and results from its implementation in the Model for Ozone and Related chemical Tracers (MOZART4), a global chemical transport model, are presented. Artificial tracers are added to the mechanism, thus, not affecting the standard chemistry. The results are linear in the troposphere, i.e., the sum of ozone from individual tagged sources equals the ozone from all sources to within $3 \%$ in zonal mean monthly averages. In addition, the tagged ozone is shown to equal the standard ozone, when all tropospheric sources are tagged and stratospheric input is turned off. The stratospheric ozone contribution to the troposphere determined from the difference between total ozone and ozone from all tagged sources is significantly less than estimates using a traditional stratospheric ozone tracer ( $8 \mathrm{vs} .20 \mathrm{ppbv}$ at the surface). The commonly used technique of perturbing NO emissions by $20 \%$ in a region to determine its ozone contribution is compared to the tagging technique, showing that the tagged ozone is $2-4$ times the ozone contribution that was deduced from perturbing emissions. The ozone tagging described here is useful for identifying source contributions based on NO emissions in a given state of the atmosphere, such as for quantifying the ozone budget.
\end{abstract}

\section{Introduction}

The transport of pollution from one region (state, country or continent) to another has been the goal of a great number of studies due to its importance to local air quality, and consequently human health and ecosystems (e.g., Dentener et al., 2010). Global chemical transport models have been used in a variety of ways to determine source attributions at given locations and source-receptor relationships. Pollutants such as carbon monoxide (CO), with relatively simple chemistry (i.e., directly emitted, lost through reaction with $\mathrm{OH}$ and dry deposition), can be easily "tagged" according to emission source types or regions for attribution studies (e.g., Granier et al., 1999; Pfister et al., 2004, 2011) or inverse modelling (e.g., Pétron et al., 2004; Arellano et al., 2006). The contribution of isoprene emissions to formaldehyde, carbon monoxide and other products was determined through a tagging technique similar to that presented here for ozone, where duplicate "tagged" species were created for each carbon compound derived from isoprene (Pfister et al., 2008a). The $\mathrm{NO}_{\mathrm{x}}$ budget was analysed in the study of Lamarque et al. (1996) by tagging each type of $\mathrm{NO}_{\mathrm{x}}$ source. For that study, the separate source tracers equaled the total $\mathrm{NO}_{\mathrm{x}}$, and the nonlinearities of loss and production rates were taken into account so that the sum of the tagged tracers equalled total $\mathrm{NO}_{\mathrm{x}}$.

Ozone, however, has quite complex chemical production and loss processes, and is not directly emitted, so identifying its source contributions requires slightly different procedures. One method of understanding source contributions to tropospheric ozone has been to set the emissions of a given region to zero and compare the results to a standard simulation with no emission perturbation to determine the influence of that region on other regions (e.g., Fiore et al., 2002; Guerova et al., 2006). Other studies have made a small change (5-20\%) in the emissions of a region, and then scaled the resulting difference from a standard run to estimate the total impact of that region. This technique has been used 
by the model comparisons performed for the Task Force on Hemispheric Transport of Air Pollution (HTAP; http://www. htap.org). Analyses of these simulations have provided estimates of foreign emissions on local ozone concentrations (e.g., Fiore et al., 2009; Reidmiller et al., 2009; Wild et al., 2012). Wu et al. (2009) showed that estimates of ozone contributions are significantly different when estimated by removing all the emissions in a source region, in comparison to making small perturbations in the emissions, due to the nonlinearity of ozone photochemistry.

A simplified $\mathrm{NO}_{\mathrm{x}}$-ozone tagging scheme, suitable for long chemistry climate simulations, has been presented by Grewe (2004). In that treatment, each $\mathrm{NO}_{\mathrm{x}}$ source produces a $\mathrm{NO}_{\mathrm{y}}$ (total reactive nitrogen) tracer in addition to the standard chemical scheme. Each $\mathrm{NO}_{\mathrm{y}}$ tracer has a corresponding ozone tracer that is produced at a fraction of the ozone production rate corresponding to the fraction of each $\mathrm{NO}_{\mathrm{y}}$ tracer to total $\mathrm{NO}_{\mathrm{y}}$. This methodology was compared to the procedure of perturbing emissions as a means of estimating source contributions by Grewe et al. (2010), with a specific application shown in Grewe et al. (2012). The contributions of $\mathrm{NO}_{\mathrm{x}}$ sources to ozone radiative forcing trends have also been estimated (Dahlmann et al., 2011).

In this paper, we present a technique for quantifying source contributions to tropospheric ozone distributions by "tagging" emissions of NO and its resulting products and following them to the production of ozone. This technique adds synthetic tracers to the chemical mechanism that do not modify the original chemistry, but make use of the mixing ratios and loss rates of the full, standard chemistry. The tagging scheme presented here has been used in several studies (Lamarque et al., 2005; Pfister et al., 2006; Hess and Lamarque, 2007; Pfister et al., 2008b; Emmons et al., 2010a; Brown-Steiner and Hess, 2011; Wespes et al., 2012), but never fully documented. We present here the details of the mechanism (in Sect. 2), and illustrate the additive qualities of the technique and comparisons to other attribution techniques (Sect. 3).

\section{Tagged mechanism}

The method we use to attribute the ozone concentration produced by a given source type or region is through the "tagging" of nitrogen (i.e., $\mathrm{NO}$ and $\mathrm{NO}_{2}$ ) emissions. Essentially a duplicate set of tracers for all compounds containing $\mathrm{N}$ are added to the mechanism; these new tracers are affected by the compounds of the full mechanism, but do not influence them. The tagged $\mathrm{NO}_{\mathrm{x}}$ is traced through all of the odd nitrogen species (e.g., $\mathrm{PAN}, \mathrm{HNO}_{3}$, organic nitrates) to account for the recycling of $\mathrm{NO}_{\mathrm{x}}$. The photolysis of tagged $\mathrm{NO}_{2}$ produces tagged $\mathrm{O}$, most of which goes on to produce tagged $\mathrm{O}_{3}$. Tagged $\mathrm{O}_{3}$ is then destroyed at the same rate as the total ozone. While ozone production also requires the presence of peroxy radicals $\left(\mathrm{HO}_{2}\right.$ and $\left.\mathrm{RO}_{2}\right)$ resulting from the
Table 1. Tagged species in the MOZART-4 tagged ozone mechanism.

\begin{tabular}{ll}
\hline Symbolic name & Atomic composition \\
\hline XNO & $\mathrm{NO}$ \\
XNO2 & $\mathrm{NO}_{2}$ \\
XNO3 & $\mathrm{NO}_{3}$ \\
XHNO3 & $\mathrm{HNO}_{3}$ \\
XHO2NO2 & $\mathrm{HNO}_{4}$ \\
XNO2NO3 & $\mathrm{N}_{2} \mathrm{O}_{5}$ \\
NO2XNO3 & $\mathrm{N}_{2} \mathrm{O}_{5}$ \\
XPAN & $\mathrm{CH}_{3} \mathrm{CO}_{3} \mathrm{NO}_{2}$ \\
XONIT & $\mathrm{CH}_{3} \mathrm{COCH}_{2} \mathrm{ONO}_{2}$ \\
XMPAN & $\mathrm{CH}_{2} \mathrm{CCH}_{3} \mathrm{CO}_{3} \mathrm{NO}_{2}$ \\
XISOPNO3 & $\mathrm{CH}_{2} \mathrm{CHCCH}_{3} \mathrm{OOCH}_{2} \mathrm{ONO}_{2}$ \\
XONITR & $\mathrm{CH}_{2} \mathrm{CCH}_{3} \mathrm{CHONO}_{2} \mathrm{CH}_{2} \mathrm{OH}$ \\
XNH4NO3 & $\mathrm{NH}_{4} \mathrm{NO}_{3}$ \\
OA & $\mathrm{O}$ \\
O1DA & $\mathrm{O}^{2}$ \\
O3A & $\mathrm{O}_{3}$ \\
\hline
\end{tabular}

oxidation of hydrocarbons, we have chosen to trace the nitrogen species for determining ozone sources.

A procedure for tracing emissions of VOC (volatile organic compounds) to peroxy radicals and then ozone has been demonstrated by Butler et al. (2011). It is similar to our NO-tagging procedure in that it adds additional tracers for the tagged compounds that do not change the original chemistry. Since ozone formation requires both NO and peroxy radicals from VOCs, either pathway could be followed for source attribution, depending on the goal of the study. Since most of the atmosphere is $\mathrm{NO}_{\mathrm{x}}$-limited, and the focus of this work was large scale attribution in a global model, tagging NO is an appropriate choice. When tagging all tropospheric contributions to ozone, tracing nitrogen compounds should give comparable results to tracing VOCs and peroxy radicals. However, if one wants to understand the impact of individual VOCs, or source sectors that might have different VOC speciation, then the Butler et al. (2011) procedure would be valuable.

Table 1 lists the additional, tagged species needed to determine the tagged ozone. The photolysis and kinetic reactions for the tagged species are listed in Tables 2 and 3 along with the corresponding reactions for the non-tagged species. The tagged $\mathrm{N}$ species are indicated by $\mathrm{XN}$, while the tagged $\mathrm{O}_{\mathrm{x}}$ species end in A (e.g., O3A). For the tagged reactions, any non-tagged species among the reactants are included as products in each reaction, so that their concentration is not affected by the tagged species. Similarly, the non-tagged products are not included in the tagged reaction. For example, the standard reaction $\mathrm{NO}+\mathrm{HO}_{2}$ produces $\mathrm{NO}_{2}+\mathrm{OH}$. In the tagged reaction, tagged $\mathrm{NO}$ goes to tagged $\mathrm{NO}_{2}$ using the current $\mathrm{HO}_{2}$ concentration, but the $\mathrm{HO}_{2}$ is unchanged by the reaction, and no additional $\mathrm{OH}$ is 
Table 2. Photolysis reactions of tagged species.

\begin{tabular}{|c|c|c|c|c|c|}
\hline \multicolumn{3}{|l|}{ Original reaction } & \multicolumn{3}{|l|}{ Tagged reaction(s) } \\
\hline $\mathrm{O} 3+h v$ & $\rightarrow$ & $\mathrm{O} 1 \mathrm{D}+\mathrm{O} 2$ & $\mathrm{O} 3 \mathrm{~A}+h v$ & $\rightarrow$ & O1DA \\
\hline $\mathrm{O} 3+h v$ & $\rightarrow$ & $\mathrm{O}+\mathrm{O} 2$ & $\mathrm{O} 3 \mathrm{~A}+h v$ & $\rightarrow$ & $\mathrm{OA}$ \\
\hline $\mathrm{NO} 2+h v$ & $\rightarrow$ & $\mathrm{NO}+\mathrm{O}$ & $\mathrm{XNO} 2+h v$ & $\rightarrow$ & $\mathrm{XNO}+\mathrm{OA}$ \\
\hline \multirow[t]{2}{*}{$\mathrm{N} 2 \mathrm{O} 5+h v$} & $\rightarrow$ & $\mathrm{NO} 2+\mathrm{NO} 3$ & $\mathrm{XNO} 2 \mathrm{NO} 3+h v$ & $\rightarrow$ & $\mathrm{XNO} 2$ \\
\hline & & & $\mathrm{NO} 2 \mathrm{XNO}_{3}+h v$ & $\rightarrow$ & $\mathrm{XNO} 3$ \\
\hline $\mathrm{HNO} 3+h v$ & $\rightarrow$ & $\mathrm{NO} 2+\mathrm{OH}$ & $\mathrm{XHNO} 3+h v$ & $\rightarrow$ & $\mathrm{XNO} 2$ \\
\hline $\mathrm{NO} 3+h v$ & $\rightarrow$ & $0.89 * \mathrm{NO} 2+0.11 * \mathrm{NO}+0.89 * \mathrm{O} 3$ & $\mathrm{XNO} 3+h v$ & $\rightarrow$ & $0.89 * \mathrm{XNO} 2+0.11 * \mathrm{XNO}+0.89 * \mathrm{O} 3 \mathrm{~A}$ \\
\hline $\mathrm{HO} 2 \mathrm{NO} 2+h v$ & $\rightarrow$ & $0.33 * \mathrm{OH}+0.33 * \mathrm{NO} 3+0.66 * \mathrm{NO} 2+0.66 * \mathrm{HO} 2$ & $\mathrm{XHO} 2 \mathrm{NO} 2+h v$ & $\rightarrow$ & $0.33 * \mathrm{XNO} 3+0.66 * \mathrm{XNO} 2$ \\
\hline $\mathrm{PAN}+h v$ & $\rightarrow$ & $0.6 * \mathrm{CH} 3 \mathrm{CO} 3+0.06 * \mathrm{NO} 2+0.4 * \mathrm{CH} 3 \mathrm{O} 2+0.4 * \mathrm{NO} 3$ & $\mathrm{XPAN}+h v$ & $\rightarrow$ & $0.6 * \mathrm{XNO} 2+0.4 * \mathrm{XNO} 3$ \\
\hline MPAN $+h v$ & $\rightarrow$ & $\mathrm{MCO} 3+\mathrm{NO} 2$ & $\mathrm{XMPAN}+h v$ & $\rightarrow$ & $\mathrm{XNO} 2$ \\
\hline ONITR $+h v$ & $\rightarrow$ & $\mathrm{HO} 2+\mathrm{CO}+\mathrm{NO} 2+\mathrm{CH} 2 \mathrm{O}$ & $\mathrm{XONITR}+h v$ & $\rightarrow$ & $\mathrm{XNO} 2$ \\
\hline
\end{tabular}
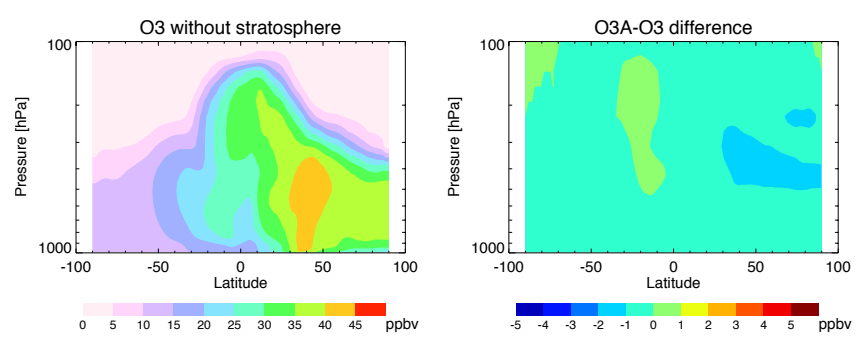

Fig. 1. Simulation test with no stratospheric ozone, for June 2008. Left: Base ozone, without stratospheric input; Right: difference between tagged ozone and base ozone.

produced (see Table 3). Therefore, oxidant levels and nontagged species are unchanged and the photochemistry occurs just as if the tagging were not included. There are a few reactions that are nonlinear in nitrogen species, such as $\mathrm{NO}_{2}+\mathrm{NO}_{3}$, so the combination of tagged and non-tagged nitrogen must be considered (as discussed in Lamarque et al., 1996). As shown in Table 3, there are two tagged reactions for $\mathrm{NO}_{2}+\mathrm{NO}_{3}+\mathrm{M} \rightarrow \mathrm{N}_{2} \mathrm{O}_{5}+\mathrm{M}$ :

$$
\begin{aligned}
& \mathrm{XNO}_{2}+\mathrm{NO}_{3}+\mathrm{M} \rightarrow \mathrm{XNO}_{2} \mathrm{NO}_{3}+\mathrm{NO}_{3}+\mathrm{M} \\
& \mathrm{NO}_{2}+\mathrm{XNO}_{3}+\mathrm{M} \rightarrow \mathrm{NO}_{2} \mathrm{XNO}_{3}+\mathrm{NO}_{2}+\mathrm{M}
\end{aligned}
$$

The tagged species are operated on by wet and dry deposition at the same rate as their non-tagged counterparts. Tagged ozone, $\mathrm{NO}$ and $\mathrm{NO}_{2}$ are relaxed to zero in the stratosphere on a timescale of ten days. MOZART-4 does not include explicit stratospheric chemistry, but constrains the mixing ratios of ozone, $\mathrm{NO}_{\mathrm{x}}$ and $\mathrm{NO}_{\mathrm{y}}$ in the stratosphere to climatologies (see Emmons et al., 2010b for details).

\section{Results}

The results presented here are from simulations of MOZART-4 (Emmons et al., 2010b) for 2008, after a 6month spin-up for each tagging simulation. The meteorological fields used to drive MOZART-4 for this study are from the
NASA Global Modelling and Assimilation Office (GMAO) GEOS-5 assimilation products (http://gmao.gsfc.nasa.gov/ products/). The emissions are the same as used in Wespes et al. (2012), with anthropogenic emissions from D. Streets' ARCTAS inventory (see http://www.cgrer.uiowa.edu/arctas/ emission.html), which is based on Zhang et al. (2009), and fire emissions from the Fire Inventory from NCAR (FINN, Wiedinmyer et al., 2011).

NO emissions are included for surface anthropogenic sources (including fossil fuel and biofuel combustion), open fire burning, from soil, lightning and aircraft. Soil emissions include a contribution from fertiliser, with the natural source calculated online based on soil moisture (cf., Emmons et al., 2010b). Lightning emissions are also calculated online in the model, using the Price et al. (1997) parameterisation dependent on cloud height (see Emmons et al., 2010b, for details). Aircraft emissions are emitted as a 3-D field with monthly variation (Emmons et al., 2010b). Table 4 gives the monthly global NO emissions for each sector. The majority of the emissions, by far, are from anthropogenic surface sources. However, away from urban areas, and in the free troposphere, the other sources can be locally very important for ozone production.

\subsection{Test of method}

To illustrate that the tagging method is sound, and has been implemented without any errors, a simulation without any stratospheric ozone transport into the troposphere has been performed. In a standard simulation, above the tropopause $\mathrm{O}_{3}, \mathrm{NO}, \mathrm{NO}_{2}$ and $\mathrm{HNO}_{3}$ are relaxed to a stratospheric climatology with a 10-day relaxation time constant. For this test simulation, both the tagged and untagged versions of $\mathrm{O}_{3}, \mathrm{NO}, \mathrm{NO}_{2}$ and $\mathrm{HNO}_{3}$ are relaxed to zero above the tropopause. The results of this simulation, where all tropospheric $\mathrm{NO}_{\mathrm{x}}$ sources have been tagged, are shown in Fig. 1 . Without any influx of stratospheric ozone to the troposphere, we expect the tagged ozone and base ozone to be nearly identical. The difference between the base and tagged ozone distributions in this test case is less than about $1 \mathrm{ppbv}$ in 
Table 3. Gas-phase reactions of tagged species.

\begin{tabular}{|c|c|c|c|c|c|}
\hline \multicolumn{3}{|l|}{ Original reaction } & \multicolumn{3}{|l|}{ Tagged reaction(s) } \\
\hline $\mathrm{O}+\mathrm{O} 2+\mathrm{M}$ & $\rightarrow$ & $\mathrm{O} 3+\mathrm{M}$ & $\mathrm{OA}+\mathrm{O} 2+\mathrm{M}$ & $\rightarrow$ & $\mathrm{O} 3 \mathrm{~A}+\mathrm{M}$ \\
\hline $\mathrm{O}+\mathrm{O} 3$ & $\rightarrow$ & $2 * \mathrm{O} 2$ & $\mathrm{OA}+\mathrm{O} 3$ & $\rightarrow$ & $\mathrm{O} 3$ \\
\hline & & & $\mathrm{O}+\mathrm{O} 3 \mathrm{~A}$ & $\rightarrow$ & $\mathrm{O}$ \\
\hline $\mathrm{O} 1 \mathrm{D}+\mathrm{N} 2$ & $\rightarrow$ & $\mathrm{O}+\mathrm{N} 2$ & $\mathrm{O} 1 \mathrm{DA}+\mathrm{N} 2$ & $\rightarrow$ & $\mathrm{OA}+\mathrm{N} 2$ \\
\hline $\mathrm{O} 1 \mathrm{D}+\mathrm{O} 2$ & $\rightarrow$ & $\mathrm{O}+\mathrm{O} 2$ & $\mathrm{O} 1 \mathrm{DA}+\mathrm{O} 2$ & $\rightarrow$ & $\mathrm{OA}+\mathrm{O} 2$ \\
\hline $\mathrm{O} 1 \mathrm{D}+\mathrm{H} 2 \mathrm{O}$ & $\rightarrow$ & $2 * \mathrm{OH}$ & $\mathrm{O} 1 \mathrm{DA}+\mathrm{H} 2 \mathrm{O}$ & $\rightarrow$ & $\mathrm{H} 2 \mathrm{O}$ \\
\hline $\mathrm{H} 2+\mathrm{O} 1 \mathrm{D}$ & $\rightarrow$ & $\mathrm{HO} 2+\mathrm{OH}$ & $\mathrm{H} 2+\mathrm{O} 1 \mathrm{DA}$ & $\rightarrow$ & $\mathrm{H} 2$ \\
\hline $\mathrm{O}+\mathrm{OH}$ & $\rightarrow$ & $\mathrm{HO} 2+\mathrm{O} 2$ & $\mathrm{OA}+\mathrm{OH}$ & $\rightarrow$ & $\mathrm{OH}$ \\
\hline $\mathrm{HO} 2+\mathrm{O}$ & $\rightarrow$ & $\mathrm{OH}+\mathrm{O} 2$ & $\mathrm{HO} 2+\mathrm{OA}$ & $\rightarrow$ & $\mathrm{HO} 2$ \\
\hline $\mathrm{OH}+\mathrm{O} 3$ & $\rightarrow$ & $\mathrm{HO} 2+\mathrm{O} 2$ & $\mathrm{OH}+\mathrm{O} 3 \mathrm{~A}$ & $\rightarrow$ & $\mathrm{OH}$ \\
\hline $\mathrm{HO} 2+\mathrm{O} 3$ & $\rightarrow$ & $\mathrm{OH}+2 * \mathrm{O} 2$ & $\mathrm{HO} 2+\mathrm{O} 3 \mathrm{~A}$ & $\rightarrow$ & $\mathrm{HO} 2$ \\
\hline $\mathrm{N} 2 \mathrm{O}+\mathrm{O} 1 \mathrm{D}$ & $\rightarrow$ & $\mathrm{N} 2+\mathrm{O} 2$ & $\mathrm{~N} 2 \mathrm{O}+\mathrm{O} 1 \mathrm{DA}$ & $\rightarrow$ & $\mathrm{N} 2 \mathrm{O}$ \\
\hline $\mathrm{N} 2 \mathrm{O}+\mathrm{O} 1 \mathrm{D}$ & $\rightarrow$ & $2 * \mathrm{NO}$ & $\mathrm{N} 2 \mathrm{O}+\mathrm{O} 1 \mathrm{DA}$ & $\rightarrow$ & $\mathrm{N} 2 \mathrm{O}$ \\
\hline $\mathrm{NO}+\mathrm{HO} 2$ & $\rightarrow$ & $\mathrm{NO} 2+\mathrm{OH}$ & $\mathrm{XNO}+\mathrm{HO} 2$ & $\rightarrow$ & $\mathrm{XNO} 2+\mathrm{HO} 2$ \\
\hline \multirow[t]{2}{*}{$\mathrm{NO}+\mathrm{O} 3$} & $\rightarrow$ & $\mathrm{NO} 2+\mathrm{O} 2$ & $\mathrm{XNO}+\mathrm{O} 3$ & $\rightarrow$ & $\mathrm{XNO} 2+\mathrm{O} 3$ \\
\hline & & & $\mathrm{NO}+\mathrm{O} 3 \mathrm{~A}$ & $\rightarrow$ & NO \\
\hline \multirow[t]{2}{*}{$\mathrm{NO} 2+\mathrm{O}$} & $\rightarrow$ & $\mathrm{NO}+\mathrm{O} 2$ & $\mathrm{XNO} 2+\mathrm{O}$ & $\rightarrow$ & $\mathrm{XNO}+\mathrm{O}$ \\
\hline & & & $\mathrm{NO} 2+\mathrm{OA}$ & $\rightarrow$ & $\mathrm{NO} 2$ \\
\hline \multirow[t]{2}{*}{$\mathrm{NO} 2+\mathrm{O} 3$} & $\rightarrow$ & $\mathrm{NO} 3+\mathrm{O} 2$ & $\mathrm{XNO} 2+\mathrm{O} 3$ & $\rightarrow$ & $\mathrm{XNO} 3+\mathrm{O} 3$ \\
\hline & & & $\mathrm{NO} 2+\mathrm{O} 3 \mathrm{~A}$ & $\rightarrow$ & $\mathrm{NO} 2$ \\
\hline $\mathrm{NO} 3+\mathrm{HO} 2$ & $\rightarrow$ & $\mathrm{OH}+\mathrm{NO} 2$ & $\mathrm{XNO} 3+\mathrm{HO} 2$ & $\rightarrow$ & $\mathrm{HO} 2+\mathrm{XNO} 2$ \\
\hline \multirow[t]{2}{*}{$\mathrm{NO} 2+\mathrm{NO} 3+\mathrm{M}$} & $\rightarrow$ & $\mathrm{N} 2 \mathrm{O} 5+\mathrm{M}$ & $\mathrm{XNO} 2+\mathrm{NO} 3+\mathrm{M}$ & $\rightarrow$ & $\mathrm{XNO} 2 \mathrm{NO} 3+\mathrm{NO} 3+\mathrm{M}$ \\
\hline & & & $\mathrm{NO} 2+\mathrm{XNO} 3+\mathrm{M}$ & $\rightarrow$ & $\mathrm{NO} 2 \mathrm{XNO}_{3}+\mathrm{NO} 2+\mathrm{M}$ \\
\hline \multirow{2}{*}{$\mathrm{N} 2 \mathrm{O} 5+\mathrm{M}$} & $\rightarrow$ & $\mathrm{NO} 2+\mathrm{NO} 3+\mathrm{M}$ & $\mathrm{XNO} 2 \mathrm{NO} 3+\mathrm{M}$ & $\rightarrow$ & $\mathrm{XNO} 2+\mathrm{M}$ \\
\hline & & & $\mathrm{NO} 2 \mathrm{XNO}_{3}+\mathrm{M}$ & $\rightarrow$ & $\mathrm{XNO} 3+\mathrm{M}$ \\
\hline $\mathrm{NO} 2+\mathrm{OH}+\mathrm{M}$ & $\rightarrow$ & $\mathrm{HNO} 3+\mathrm{M}$ & $\mathrm{XNO} 2+\mathrm{OH}+\mathrm{M}$ & $\rightarrow$ & $\mathrm{XHNO} 3+\mathrm{OH}+\mathrm{M}$ \\
\hline $\mathrm{HNO} 3+\mathrm{OH}$ & $\rightarrow$ & $\mathrm{NO} 3+\mathrm{H} 2 \mathrm{O}$ & $\mathrm{XHNO} 3+\mathrm{OH}$ & $\rightarrow$ & $\mathrm{XNO} 3+\mathrm{OH}$ \\
\hline \multirow[t]{2}{*}{$\mathrm{NO} 3+\mathrm{NO}$} & $\rightarrow$ & $2 * \mathrm{NO} 2$ & $\mathrm{XNO} 3+\mathrm{NO}$ & $\rightarrow$ & $\mathrm{XNO} 2+\mathrm{NO}$ \\
\hline & & & $\mathrm{NO} 3+\mathrm{XNO}$ & $\rightarrow$ & $\mathrm{XNO} 2+\mathrm{NO} 3$ \\
\hline $\mathrm{NO} 2+\mathrm{HO} 2+\mathrm{M}$ & $\rightarrow$ & $\mathrm{HO} 2 \mathrm{NO} 2+\mathrm{M}$ & $\mathrm{XNO} 2+\mathrm{HO} 2+\mathrm{M}$ & $\rightarrow$ & $\mathrm{XHO} 2 \mathrm{NO} 2+\mathrm{HO} 2+\mathrm{M}$ \\
\hline $\mathrm{HO} 2 \mathrm{NO} 2+\mathrm{OH}$ & $\rightarrow$ & $\mathrm{H} 2 \mathrm{O}+\mathrm{NO} 2+\mathrm{O} 2$ & $\mathrm{XHO} 2 \mathrm{NO} 2+\mathrm{OH}$ & $\rightarrow$ & $\mathrm{XNO} 2+\mathrm{OH}$ \\
\hline $\mathrm{HO} 2 \mathrm{NO} 2+\mathrm{M}$ & $\rightarrow$ & $\mathrm{HO} 2+\mathrm{NO} 2+\mathrm{M}$ & $\mathrm{XHO} 2 \mathrm{NO} 2+\mathrm{M}$ & $\rightarrow$ & $\mathrm{XNO} 2+\mathrm{M}$ \\
\hline $\mathrm{CH} 4+\mathrm{O} 1 \mathrm{D}$ & $\rightarrow$ & $0.75^{*} \mathrm{CH} 3 \mathrm{O} 2+0.75 * \mathrm{OH}+0.25 * \mathrm{CH} 2 \mathrm{O}+0.4 * \mathrm{HO} 2+0.05 * \mathrm{H} 2$ & $\mathrm{CH} 4+\mathrm{O} 1 \mathrm{DA}$ & $\rightarrow$ & $\mathrm{CH} 4$ \\
\hline $\mathrm{CH} 3 \mathrm{O} 2+\mathrm{NO}$ & $\rightarrow$ & $\mathrm{CH} 2 \mathrm{O}+\mathrm{NO} 2+\mathrm{HO} 2$ & $\mathrm{CH} 3 \mathrm{O} 2+\mathrm{XNO}$ & $\rightarrow$ & $\mathrm{CH} 3 \mathrm{O} 2+\mathrm{XNO} 2$ \\
\hline $\mathrm{CH} 2 \mathrm{O}+\mathrm{NO} 3$ & $\rightarrow$ & $\mathrm{CO}+\mathrm{HO} 2+\mathrm{HNO} 3$ & $\mathrm{CH} 2 \mathrm{O}+\mathrm{XNO} 3$ & $\rightarrow$ & $\mathrm{CH} 2 \mathrm{O}+\mathrm{XHNO} 3$ \\
\hline $\mathrm{C} 2 \mathrm{H} 4+\mathrm{O} 3$ & $\rightarrow$ & $\mathrm{CH} 2 \mathrm{O}+0.12 * \mathrm{HO} 2+0.5 * \mathrm{CO}+0.12 * \mathrm{OH}+0.25 * \mathrm{CH} 3 \mathrm{COOH}$ & $\mathrm{C} 2 \mathrm{H} 4+\mathrm{O} 3 \mathrm{~A}$ & $\rightarrow$ & $\mathrm{C} 2 \mathrm{H} 4$ \\
\hline $\mathrm{EO} 2+\mathrm{NO}$ & $\rightarrow$ & $\mathrm{EO}+\mathrm{NO} 2$ & $\mathrm{EO} 2+\mathrm{XNO}$ & $\rightarrow$ & $\mathrm{EO} 2+\mathrm{XNO} 2$ \\
\hline $\mathrm{C} 2 \mathrm{H} 5 \mathrm{O} 2+\mathrm{NO}$ & $\rightarrow$ & $\mathrm{CH} 3 \mathrm{CHO}+\mathrm{HO} 2+\mathrm{NO} 2$ & $\mathrm{C} 2 \mathrm{H} 5 \mathrm{O} 2+\mathrm{XNO}$ & $\rightarrow$ & $\mathrm{C} 2 \mathrm{H} 5 \mathrm{O} 2+\mathrm{XNO} 2$ \\
\hline $\mathrm{CH} 3 \mathrm{CHO}+\mathrm{NO} 3$ & $\rightarrow$ & $\mathrm{CH} 3 \mathrm{CO} 3+\mathrm{HNO} 3$ & $\mathrm{CH} 3 \mathrm{CHO}+\mathrm{XNO} 3$ & $\rightarrow$ & $\mathrm{CH} 3 \mathrm{CHO}+\mathrm{XHNO} 3$ \\
\hline $\mathrm{CH} 3 \mathrm{CO} 3+\mathrm{NO}$ & $\rightarrow$ & $\mathrm{CH} 3 \mathrm{O} 2+\mathrm{NO} 2$ & $\mathrm{CH} 3 \mathrm{CO} 3+\mathrm{XNO}$ & $\rightarrow$ & $\mathrm{CH} 3 \mathrm{CO} 3+\mathrm{XNO} 2$ \\
\hline $\mathrm{CH} 3 \mathrm{CO} 3+\mathrm{NO} 2+\mathrm{M}$ & $\rightarrow$ & $\mathrm{PAN}+\mathrm{M}$ & $\mathrm{CH} 3 \mathrm{CO} 3+\mathrm{XNO} 2+\mathrm{M}$ & $\rightarrow$ & $\mathrm{XPAN}+\mathrm{CH} 3 \mathrm{CO} 3+\mathrm{M}$ \\
\hline $\mathrm{PAN}+\mathrm{OH}$ & $\rightarrow$ & $\mathrm{CH} 2 \mathrm{O}+\mathrm{NO} 3$ & $\mathrm{XPAN}+\mathrm{OH}$ & $\rightarrow$ & $\mathrm{XNO} 3+\mathrm{OH}$ \\
\hline $\mathrm{PAN}+\mathrm{M}$ & $\rightarrow$ & $\mathrm{CH} 3 \mathrm{CO} 3+\mathrm{NO} 2+\mathrm{M}$ & $\mathrm{XPAN}+\mathrm{M}$ & $\rightarrow$ & $\mathrm{XNO} 2+\mathrm{M}$ \\
\hline $\mathrm{C} 3 \mathrm{H} 6+\mathrm{O} 3$ & $\rightarrow$ & $\begin{array}{l}0.54 * \mathrm{CH} 2 \mathrm{O}+0.19 * \mathrm{HO} 2+0.33 * \mathrm{OH}+0.5 * \mathrm{CH} 3 \mathrm{CHO}+0.56 * \mathrm{CO} \\
+0.31 * \mathrm{CH} 3 \mathrm{O} 2+.25 * \mathrm{CH} 3 \mathrm{COOH}+0.08 * \mathrm{CH} 4\end{array}$ & $\mathrm{C} 3 \mathrm{H} 6+\mathrm{O} 3 \mathrm{~A}$ & $\rightarrow$ & $\mathrm{C} 3 \mathrm{H} 6$ \\
\hline $\mathrm{C} 3 \mathrm{H} 6+\mathrm{NO} 3$ & $\rightarrow$ & ONIT & $\mathrm{C} 3 \mathrm{H} 6+\mathrm{XNO} 3$ & $\rightarrow$ & $\mathrm{C} 3 \mathrm{H} 6+\mathrm{XONIT}$ \\
\hline $\mathrm{PO} 2+\mathrm{NO}$ & $\rightarrow$ & $\mathrm{CH} 3 \mathrm{CHO}+\mathrm{CH} 2 \mathrm{O}+\mathrm{HO} 2+\mathrm{NO} 2$ & $\mathrm{PO} 2+\mathrm{XNO}$ & $\rightarrow$ & $\mathrm{PO} 2+\mathrm{XNO} 2$ \\
\hline $\mathrm{C} 3 \mathrm{H} 7 \mathrm{O} 2+\mathrm{NO}$ & $\rightarrow$ & $0.82 * \mathrm{CH} 3 \mathrm{COCH} 3+\mathrm{NO} 2+0.27 * \mathrm{CH} 3 \mathrm{CHO}+\mathrm{HO} 2$ & $\mathrm{C} 3 \mathrm{H} 7 \mathrm{O} 2+\mathrm{XNO}$ & $\rightarrow$ & $\mathrm{C} 3 \mathrm{H} 7 \mathrm{O} 2+\mathrm{XNO} 2$ \\
\hline $\mathrm{RO} 2+\mathrm{NO}$ & $\rightarrow$ & $\mathrm{CH} 3 \mathrm{CO} 3+\mathrm{CH} 2 \mathrm{O}+\mathrm{NO} 2$ & $\mathrm{RO} 2+\mathrm{XNO}$ & $\rightarrow$ & $\mathrm{RO} 2+\mathrm{XNO} 2$ \\
\hline $\mathrm{ONIT}+\mathrm{OH}$ & $\rightarrow$ & $\mathrm{NO} 2+\mathrm{CH} 3 \mathrm{COCHO}$ & $\mathrm{XONIT}+\mathrm{OH}$ & $\rightarrow$ & $\mathrm{XNO} 2+\mathrm{OH}$ \\
\hline $\mathrm{CH} 3 \mathrm{COCHO}+\mathrm{NO} 3$ & $\rightarrow$ & $\mathrm{HNO} 3+\mathrm{CO}+\mathrm{CH} 3 \mathrm{CO} 3$ & $\mathrm{CH} 3 \mathrm{COCHO}+\mathrm{XNO} 3$ & $\rightarrow$ & $\mathrm{XHNO} 3+\mathrm{CH} 3 \mathrm{COCHO}$ \\
\hline $\mathrm{ENEO} 2+\mathrm{NO}$ & $\rightarrow$ & $\mathrm{CH} 3 \mathrm{CHO}+0.5 * \mathrm{CH} 2 \mathrm{O}+0.5 * \mathrm{CH} 3 \mathrm{COCH} 3+\mathrm{HO} 2+\mathrm{NO} 2$ & $\mathrm{ENEO} 2+\mathrm{XNO}$ & $\rightarrow$ & $\mathrm{ENEO} 2+\mathrm{XNO} 2$ \\
\hline $\mathrm{MEKO} 2+\mathrm{NO}$ & $\rightarrow$ & $\mathrm{CH} 3 \mathrm{CO} 3+\mathrm{CH} 3 \mathrm{CHO}+\mathrm{NO} 2$ & $\mathrm{MEKO} 2+\mathrm{XNO}$ & $\rightarrow$ & $\mathrm{MEKO} 2+\mathrm{XNO} 2$ \\
\hline $\mathrm{MPAN}+\mathrm{OH}+\mathrm{M}$ & $\rightarrow$ & $.5 * \mathrm{HYAC}+0.5^{*} \mathrm{NO} 3+0.5^{*} \mathrm{CH} 2 \mathrm{O}+0.5 * \mathrm{HO} 2+\mathrm{M}$ & $\mathrm{XMPAN}+\mathrm{OH}+\mathrm{M}$ & $\rightarrow$ & $0.5 * \mathrm{XNO} 3+\mathrm{OH}+\mathrm{M}$ \\
\hline $\mathrm{ALKO} 2+\mathrm{NO}$ & $\rightarrow$ & $\begin{array}{l}0.4 * \mathrm{CH} 3 \mathrm{CHO}+0.1 * \mathrm{CH} 2 \mathrm{O}+0.25 * \mathrm{CH} 3 \mathrm{COCH} 3+0.9 * \mathrm{HO} 2 \\
+0.75 * \mathrm{MEK}+0.9 * \mathrm{NO} 2+0.1 * \mathrm{ONIT}\end{array}$ & $\mathrm{ALKO} 2+\mathrm{XNO}$ & $\rightarrow$ & $\begin{array}{l}\text { ALKO } 2+0.9 * \text { XNO } 2 \\
+0.1 * \text { XONIT }\end{array}$ \\
\hline $\mathrm{ISOP}+\mathrm{O} 3$ & $\rightarrow$ & $\begin{array}{l}0.4 * \mathrm{MACR}+0.2 * \mathrm{MVK}+0.07 * \mathrm{C} 3 \mathrm{H} 6+0.27 * \mathrm{OH}+0.06 * \mathrm{HO} 2 \\
+0.6 * \mathrm{CH} 2 \mathrm{O}+0.3 * \mathrm{CO}+0.1 * \mathrm{O} 3+0.2 * \mathrm{MCO} 3+0.2 * \mathrm{CH} 3 \mathrm{COOH}\end{array}$ & $\mathrm{ISOP}+\mathrm{O} 3 \mathrm{~A}$ & $\rightarrow$ & $\mathrm{ISOP}+0.1 * \mathrm{O} 3 \mathrm{~A}$ \\
\hline $\mathrm{ISOPO} 2+\mathrm{NO}$ & $\rightarrow$ & $\begin{array}{l}0.08 * \mathrm{ONITR}+0.92 * \mathrm{NO} 2+\mathrm{HO} 2+0.55 * \mathrm{CH} 2 \mathrm{O}+0.23 * \mathrm{MACR} \\
+.32 * \mathrm{MVK}+.37 * \mathrm{HYDRALD}\end{array}$ & $\mathrm{ISOPO} 2+\mathrm{XNO}$ & $\rightarrow$ & $\begin{array}{l}\text { ISOPO } 2+0.08 * \text { XONITR } \\
+0.92 * \text { XNO } 2\end{array}$ \\
\hline $\mathrm{ISOPO} 2+\mathrm{NO} 3$ & $\rightarrow$ & $\begin{array}{l}\mathrm{HO} 2+\mathrm{NO} 2+0.6 * \mathrm{CH} 2 \mathrm{O}+0.25 * \mathrm{MACR}+0.35 * \mathrm{MVK} \\
+0.4 * \mathrm{HYDRALD}\end{array}$ & $\mathrm{ISOPO} 2+\mathrm{XNO} 3$ & $\rightarrow$ & $\mathrm{ISOPO} 2+\mathrm{XNO} 2$ \\
\hline
\end{tabular}


Table 3. Continued.

\begin{tabular}{|c|c|c|c|c|c|}
\hline \multicolumn{3}{|l|}{ Original reaction } & \multicolumn{3}{|l|}{ Tagged reaction(s) } \\
\hline $\mathrm{ISOP}+\mathrm{NO} 3$ & $\rightarrow$ & ISOPNO3 & $\mathrm{ISOP}+\mathrm{XNO} 3$ & $\rightarrow$ & ISOP + XISOPNO3 \\
\hline \multirow[t]{2}{*}{ ISOPNO3 + NO } & $\rightarrow$ & $1.206 * \mathrm{NO} 2+0.072 * \mathrm{CH} 2 \mathrm{O}+0.167 * \mathrm{MACR}$ & XISOPNO3 + NO & $\rightarrow$ & $0.206 *$ XNO $2+0.794 *$ XONITR + NO \\
\hline & & $+0.039 * \mathrm{MVK}+0.794 * \mathrm{ONITR}+0.794 * \mathrm{HO} 2$ & ISOPNO3 + XNO & $\rightarrow$ & $1.000 * \mathrm{XNO} 2+\mathrm{ISOPNO} 3$ \\
\hline \multirow[t]{2}{*}{ ISOPNO3 + NO3 } & $\rightarrow$ & $1.206 * \mathrm{NO} 2+0.072 * \mathrm{CH} 2 \mathrm{O}+.167 * \mathrm{MACR}$ & XISOPNO3 + NO3 & $\rightarrow$ & $0.206 * \mathrm{XNO} 2+0.794 * \mathrm{XONITR}+\mathrm{NO} 3$ \\
\hline & & $+0.039 * \mathrm{MVK}+0.794 * \mathrm{ONITR}+0.794 * \mathrm{HO} 2$ & ISOPNO3 + XNO3 & $\rightarrow$ & $1.000 * \mathrm{XNO} 2+\mathrm{ISOPNO} 3$ \\
\hline ISOPNO3 + HO2 & $\rightarrow$ & $\begin{array}{l}0.206 * \mathrm{NO} 2+0.008 * \mathrm{CH} 2 \mathrm{O}+0.167 * \mathrm{MACR}+ \\
0.039 * \mathrm{MVK}+0.794 * \mathrm{ONITR}+0.794 * \mathrm{HO} 2\end{array}$ & ISOPNO3 + $\mathrm{HO} 2$ & $\rightarrow$ & $0.206 * \mathrm{XNO} 2+\mathrm{ISOPNO} 3$ \\
\hline $\mathrm{MVK}+\mathrm{O} 3$ & $\rightarrow$ & $\begin{array}{l}0.8 * \mathrm{CH} 2 \mathrm{O}+0.95 * \mathrm{CH} 3 \mathrm{COCHO}+0.08 * \mathrm{OH} \\
+0.2 * \mathrm{O} 3+0.06 * \mathrm{HO} 2+0.05^{*} \mathrm{CO}+0.04 * \mathrm{CH} 3 \mathrm{CHO}\end{array}$ & $\mathrm{MVK}+\mathrm{O} 3 \mathrm{~A}$ & $\rightarrow$ & $\mathrm{MVK}+0.2 * \mathrm{O} 3 \mathrm{~A}$ \\
\hline $\mathrm{MACR}+\mathrm{O} 3$ & $\rightarrow$ & $\begin{array}{l}0.8 * \mathrm{CH} 3 \mathrm{COCHO}+0.275^{*} \mathrm{HO} 2+0.2 * \mathrm{CO} \\
+0.2 * \mathrm{O} 3+0.7 * \mathrm{CH} 2 \mathrm{O}+0.215^{*} \mathrm{OH}\end{array}$ & $\mathrm{MACR}+\mathrm{O} 3 \mathrm{~A}$ & $\rightarrow$ & $\mathrm{MACR}+0.2 * \mathrm{O} 3 \mathrm{~A}$ \\
\hline $\mathrm{MACRO} 2+\mathrm{NO}$ & $\rightarrow$ & $\begin{array}{l}\mathrm{NO} 2+0.47 * \mathrm{HO} 2+0.25 * \mathrm{CH} 2 \mathrm{O}+0.25 * \mathrm{CH} 3 \mathrm{COCHO} \\
+0.53 * \mathrm{CH} 3 \mathrm{CO} 3+0.53 * \mathrm{GLYALD}+0.22 * \mathrm{HYAC} \\
+0.22 * \mathrm{CO}\end{array}$ & $\mathrm{MACRO} 2+\mathrm{XNO}$ & $\rightarrow$ & $\mathrm{XNO} 2+\mathrm{MACRO} 2$ \\
\hline $\mathrm{MACRO} 2+\mathrm{NO}$ & $\rightarrow$ & $0.8 *$ ONITR & $\mathrm{MACRO} 2+\mathrm{XNO}$ & $\rightarrow$ & $0.8^{*} \mathrm{XONITR}+\mathrm{MACRO} 2$ \\
\hline $\mathrm{MACRO} 2+\mathrm{NO} 3$ & $\rightarrow$ & $\begin{array}{l}\mathrm{NO} 2+0.53 * \mathrm{GLYALD}+0.22 * \mathrm{HYAC}+0.53 * \mathrm{CH} 3 \mathrm{CO} 3 \\
+0.25 * \mathrm{CH} 2 \mathrm{O}+0.22 * \mathrm{CO}+.25 * \mathrm{CH} 3 \mathrm{COCHO} \\
+0.47 * \mathrm{HO} 2\end{array}$ & $\mathrm{MACRO} 2+\mathrm{XNO} 3$ & $\rightarrow$ & $\mathrm{XNO} 2+\mathrm{MACRO} 2$ \\
\hline $\mathrm{MCO} 3+\mathrm{NO}$ & $\rightarrow$ & $\mathrm{NO} 2+\mathrm{CH} 2 \mathrm{O}+\mathrm{CH} 3 \mathrm{CO} 3$ & $\mathrm{MCO} 3+\mathrm{XNO}$ & $\rightarrow$ & $\mathrm{XNO} 2+\mathrm{MCO} 3$ \\
\hline $\mathrm{MCO} 3+\mathrm{NO} 3$ & $\rightarrow$ & $\mathrm{NO} 2+\mathrm{CH} 2 \mathrm{O}+\mathrm{CH} 3 \mathrm{CO} 3$ & $\mathrm{MCO} 3+\mathrm{NO} 3$ & $\rightarrow$ & $\mathrm{XNO} 2+\mathrm{MCO} 3$ \\
\hline $\mathrm{MCO} 3+\mathrm{NO} 2+\mathrm{M}$ & $\rightarrow$ & $\mathrm{MPAN}+\mathrm{M}$ & $\mathrm{MCO} 3+\mathrm{XNO} 2+\mathrm{M}$ & $\rightarrow$ & $\mathrm{XMPAN}+\mathrm{MCO} 3+\mathrm{M}$ \\
\hline $\mathrm{MPAN}+\mathrm{M}$ & $\rightarrow$ & $\mathrm{MCO} 3+\mathrm{NO} 2+\mathrm{M}$ & $\mathrm{XMPAN}+\mathrm{M}$ & $\rightarrow$ & $\mathrm{XNO} 2+\mathrm{M}$ \\
\hline $\mathrm{ONITR}+\mathrm{OH}$ & $\rightarrow$ & HYDRALD + 0.4*NO2 + $\mathrm{HO} 2$ & $\mathrm{XONITR}+\mathrm{OH}$ & $\rightarrow$ & $0.4 * \mathrm{XNO} 2+\mathrm{OH}$ \\
\hline \multirow[t]{2}{*}{$\mathrm{ONITR}+\mathrm{NO} 3$} & $\rightarrow$ & $\mathrm{HYDRALD}+\mathrm{NO} 2+\mathrm{HO} 2$ & $\mathrm{XONITR}+\mathrm{NO} 3$ & $\rightarrow$ & $0.5^{*} \mathrm{XNO} 2+\mathrm{NO} 3$ \\
\hline & & & ONITR + XNO3 & $\rightarrow$ & $0.5 * \mathrm{XNO} 2+$ ONITR \\
\hline $\mathrm{XO} 2+\mathrm{NO}$ & $\rightarrow$ & $\mathrm{NO} 2+1.5 * \mathrm{HO} 2+\mathrm{CO}+0.25 * \mathrm{CH} 3 \mathrm{COCHO}+0.25 * \mathrm{HYAC}+0.25 *$ GLYALD & $\mathrm{XO} 2+\mathrm{XNO}$ & $\rightarrow$ & $\mathrm{XNO} 2+\mathrm{XO} 2$ \\
\hline $\mathrm{XO} 2+\mathrm{NO} 3$ & $\rightarrow$ & $\mathrm{NO} 2+1.5 * \mathrm{HO} 2+\mathrm{CO}+0.25 * \mathrm{CH} 3 \mathrm{COCHO}+0.25 * \mathrm{HYAC}+0.25 * \mathrm{GLYALD}$ & $\mathrm{XO} 2+\mathrm{XNO} 3$ & $\rightarrow$ & $\mathrm{XNO} 2+\mathrm{XO} 2$ \\
\hline $\mathrm{XOH}+\mathrm{NO} 2$ & $\rightarrow$ & $0.7 * \mathrm{NO} 2+0.7 * \mathrm{BIGALD}+0.7 * \mathrm{HO} 2$ & $\mathrm{XOH}+\mathrm{XNO} 2$ & $\rightarrow$ & $\mathrm{XOH}+0.7^{*} \mathrm{XNO} 2$ \\
\hline $\mathrm{TOLO} 2+\mathrm{NO}$ & $\rightarrow$ & $0.45^{*} \mathrm{GLYOXAL}+0.45^{*} \mathrm{CH} 3 \mathrm{COCHO}+0.9 * \mathrm{BIGALD}+0.9 * \mathrm{NO} 2+0.9^{*} \mathrm{HO} 2$ & $\mathrm{TOLO} 2+\mathrm{XNO}$ & $\rightarrow$ & $\mathrm{TOLO} 2+0.9^{*} \mathrm{NO} 2$ \\
\hline $\mathrm{C} 10 \mathrm{H} 16+\mathrm{O} 3$ & $\rightarrow$ & $0.7 * \mathrm{OH}+\mathrm{MVK}+\mathrm{MACR}+\mathrm{HO} 2$ & $\mathrm{C} 10 \mathrm{H} 16+\mathrm{O} 3 \mathrm{~A}$ & $\rightarrow$ & $\mathrm{C} 10 \mathrm{H} 16$ \\
\hline $\mathrm{C} 10 \mathrm{H} 16+\mathrm{NO} 3$ & $\rightarrow$ & $\mathrm{TERPO} 2+\mathrm{NO} 2$ & $\mathrm{C} 10 \mathrm{H} 16+\mathrm{XNO} 3$ & $\rightarrow$ & $\mathrm{C} 10 \mathrm{H} 16+\mathrm{XNO} 2$ \\
\hline TERPO $2+\mathrm{NO}$ & $\rightarrow$ & $0.1 * \mathrm{CH} 3 \mathrm{COCH} 3+\mathrm{HO} 2+\mathrm{MVK}+\mathrm{MACR}+\mathrm{NO} 2$ & TERPO2 + XNO & $\rightarrow$ & TERPO $2+\mathrm{XNO} 2$ \\
\hline $\mathrm{DMS}+\mathrm{NO} 3$ & $\rightarrow$ & $\mathrm{SO} 2+\mathrm{HNO} 3$ & $\mathrm{DMS}+\mathrm{XNO} 3$ & $\rightarrow$ & DMS + XHNO3 \\
\hline \multirow[t]{2}{*}{$\mathrm{N} 2 \mathrm{O} 5$} & $\rightarrow$ & HNO3 (het) & $\mathrm{XNO} 2 \mathrm{NO} 3$ & $\rightarrow$ & XHNO3 (het) \\
\hline & & & $\mathrm{NO} 2 \mathrm{XNO}$ & $\rightarrow$ & XHNO3 (het) \\
\hline NO3 & $\rightarrow$ & HNO3 (het) & XNO3 & $\rightarrow$ & XHNO3 (het) \\
\hline NO2 & $\rightarrow$ & $0.5^{*} \mathrm{NO}+0.5^{*} \mathrm{HNO} 3$ (het) & $\mathrm{XNO} 2$ & $\rightarrow$ & $0.5 * \mathrm{XNO}+0.5 * \mathrm{XHNO} 3$ (het) \\
\hline
\end{tabular}

Table 4. NO emissions used in this study (global totals in $\mathrm{Tg} \mathrm{yr}^{-1}$ ).

\begin{tabular}{|c|c|c|c|c|c|c|c|c|c|c|c|c|c|}
\hline Sector & Jan & Feb & Mar & Apr & May & Jun & Jul & Aug & Sep & Oct & Nov & Dec & Annual Avg \\
\hline Anthropogenic & 69.9 & 69.9 & 69.9 & 69.9 & 69.9 & 69.9 & 69.9 & 69.9 & 69.9 & 69.9 & 69.9 & 69.9 & 69.9 \\
\hline Fires & 3.4 & 4.4 & 6.4 & 9.0 & 3.5 & 4.5 & 6.1 & 7.8 & 7.9 & 4.1 & 2.9 & 2.6 & 5.2 \\
\hline Soil & 7.3 & 6.9 & 8.4 & 10.5 & 13.1 & 14.2 & 15.5 & 14.6 & 11.6 & 9.6 & 7.9 & 7.3 & 10.6 \\
\hline Lightning & 6.2 & 5.8 & 5.8 & 6.4 & 6.6 & 7.0 & 7.3 & 7.8 & 7.0 & 6.4 & 6.0 & 5.8 & 6.5 \\
\hline Aircraft & 1.3 & 1.3 & 1.3 & 1.3 & 1.3 & 1.4 & 1.4 & 1.4 & 1.4 & 1.4 & 1.3 & 1.3 & 1.3 \\
\hline Total & 88.1 & 88.3 & 91.8 & 97.1 & 94.4 & 97 & 100.2 & 101.5 & 97.8 & 91.4 & 88 & 86.9 & 93.5 \\
\hline
\end{tabular}

the zonal average. The slight difference is due to the small amount of ozone formed directly without $\mathrm{NO}_{\mathrm{x}}$ catalysis in the oxidation of hydrocarbons (Emmons et al., 2010b).

\subsection{Tropospheric sources}

Figures 2 and 3 show maps of the tropospheric column of each tagged ozone contribution (anthropogenic surface sources, fires, soil, lightning, aircraft and the stratosphere) for January and July 2008. For this analysis, we use a chemical tropopause defined as the altitude where ozone reaches $150 \mathrm{ppbv}$ (e.g., Stevenson et al., 2006). The surface anthropogenic emissions are the largest contribution in the Northern Hemisphere in both seasons, while lightning is the dominant source in the Tropics. The location of fire emissions changes with season, with the savanna region of northern Tropical Africa having the strongest contribution in January. In July, the tropical regions of South America and Africa have notable ozone contributions from agricultural fires, with the large wildfires in Siberia and Canada also evident. The contribution from soil NO emissions are most apparent in the summer season of each hemisphere, while the stratospheric contribution is largest in winter.

Figures 4 and 5 show the vertical distribution of ozone and the tagged contributions in zonal averages for January and July, respectively. Each panel shows the location of the $150 \mathrm{ppbv}$ contour of ozone (used for determining the 
Tagged O3 Column (O3<150 ppbv) - Jan
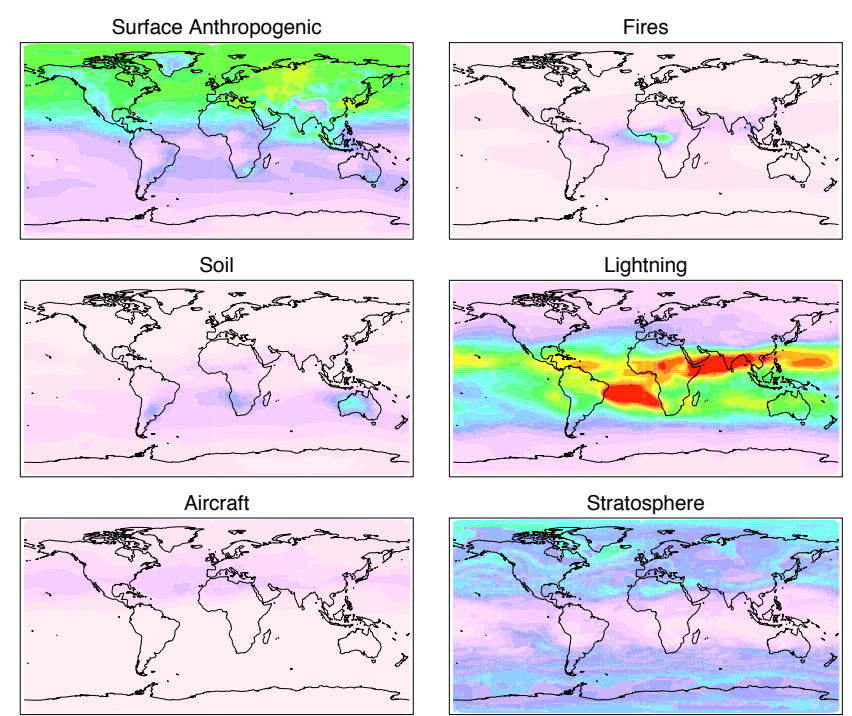

Lightning

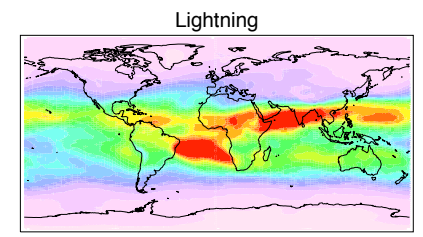

Stratosphere

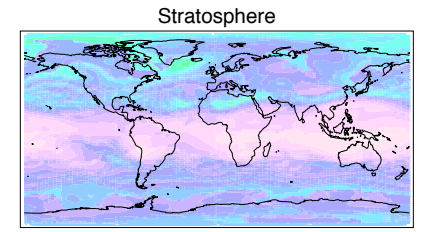

$\begin{array}{llllllllllllllllllllll}0 & 1 & 2 & 3 & 4 & 5 & 6 & 7 & 8 & 9 & 10 & 11 & 12 & 13 & 14 & 15 & 16 & 17 & 18 & 19 & 20 & \text { DU }\end{array}$

Fig. 2. Tropospheric columns of each tagged ozone source for January 2008. Stratospheric contribution is determined as the difference between total ozone and tagged ozone from all tropospheric sources. The chemical tropopause of $\mathrm{O}_{3}$ at $150 \mathrm{ppbv}$ is used.

tropospheric columns in the previous figures). In January, anthropogenic surface sources are maximum in the lower and middle troposphere of the Northern Hemisphere $(\mathrm{NH})$, while in July strong summertime convection distributes its ozone contribution throughout the troposphere at mid-latitudes. A similar summer-winter pattern is seen in the Southern Hemisphere (SH), but the anthropogenic sources are so much smaller there, the anthropogenic ozone contribution is much less. The vertical distribution of the ozone contributions from fires and soil emissions shows the seasonal variation in both the sources and convection and transport patterns. Lightning and aircraft emissions originate in the upper troposphere, with aircraft activity largest in the $\mathrm{NH}$ and lightning peaking in the tropics and in summer at mid-latitudes. While lightning produces only NO (at least in the model), sufficient concentrations of $\mathrm{CO}$ and VOCs are transported within the convective systems to lead to large amounts of ozone production (e.g., Hauglustaine et al., 2001). Aircraft have very low $\mathrm{CO}$ and hydrocarbon emissions coincident with NO, but background levels of $\mathrm{CO}$ and VOCs are sufficient for ozone production in the upper troposphere, resulting in up to $30 \mathrm{ppbv}$ ozone contribution in the zonal average in July. The stratospheric ozone contribution clearly shows the level of the tropopause approximately at the $100 \mathrm{ppbv}$ contour, with highest values in the troposphere in the winter hemisphere.

Figure 6 (top row) shows zonal averages for January and July of the contributions of ozone at the surface from each NO source type. As seen in the previous figures,
Tagged O3 Column (O3 $<150$ ppbv) - Jul
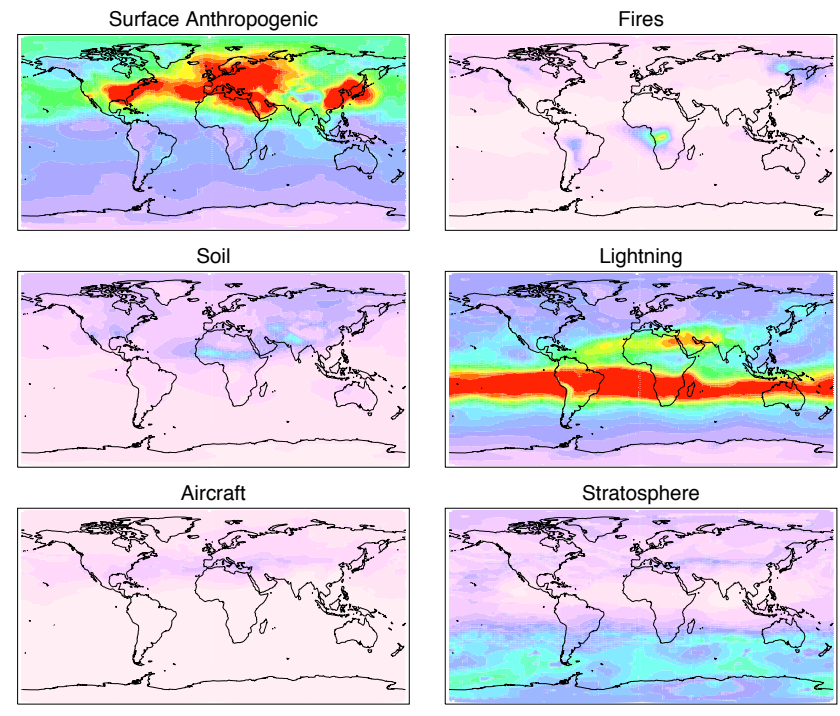

$\begin{array}{lllllllllllllllllllllll}0 & 1 & 2 & 3 & 4 & 5 & 6 & 7 & 8 & 9 & 10 & 11 & 12 & 13 & 14 & 15 & 16 & 17 & 18 & 19 & 20 & \text { DU }\end{array}$

Fig. 3. As Fig. 2, for July 2008.

the anthropogenic contribution is dominant in the Northern Hemisphere for both summer and winter. In the Southern Hemisphere, lightning and stratospheric air are a larger fraction of the total ozone. Biomass burning has a large seasonal variation, however, with a relatively small contribution to the total. Soil emissions peak in the summer mid-latitudes of both hemispheres. The bottom row of Fig. 6 shows the ozone amounts for different sources at $400 \mathrm{hPa}$, with very different relative contributions than at the surface. Lightning is the largest contribution in the Tropics and sub-Tropics. The stratospheric contribution is most significant in $\mathrm{NH}$ winter and spring. Anthropogenic emissions are the source for twice as much ozone in the $\mathrm{NH}$ than the $\mathrm{SH}$, but contribute a smaller fraction than at the surface. The small white area between the contributions from lightning and the stratosphere indicates the difference between the sum of the individually tagged sources and the result from tagging all sources at once. This shows the level of nonlinearity in the method and corresponds to $3 \%$ or less in the zonal average at $400 \mathrm{hPa}$, and less than $1 \%$ at the surface.

The same source contributions to $\mathrm{NO}_{\mathrm{x}}, \mathrm{PAN}, \mathrm{HNO}_{3}$ and total $\mathrm{NO}_{\mathrm{y}}$ at $400 \mathrm{hPa}$ are shown in Fig. 7 for July. As for ozone, the most important contributions are from anthropogenic and lightning emissions for all three of these species. The stratospheric source for these compounds is determined in the same manner as for ozone (the difference between simulated total of the species and the contribution from all tropospheric sources tagged). For $\mathrm{NO}_{\mathrm{x}}$, the stratospheric contribution is barely noticeable in these plots, but for $\mathrm{HNO}_{3}$ it is important, and for the $\mathrm{SH}$ winter is essentially the only source south of $50^{\circ} \mathrm{S}$. 

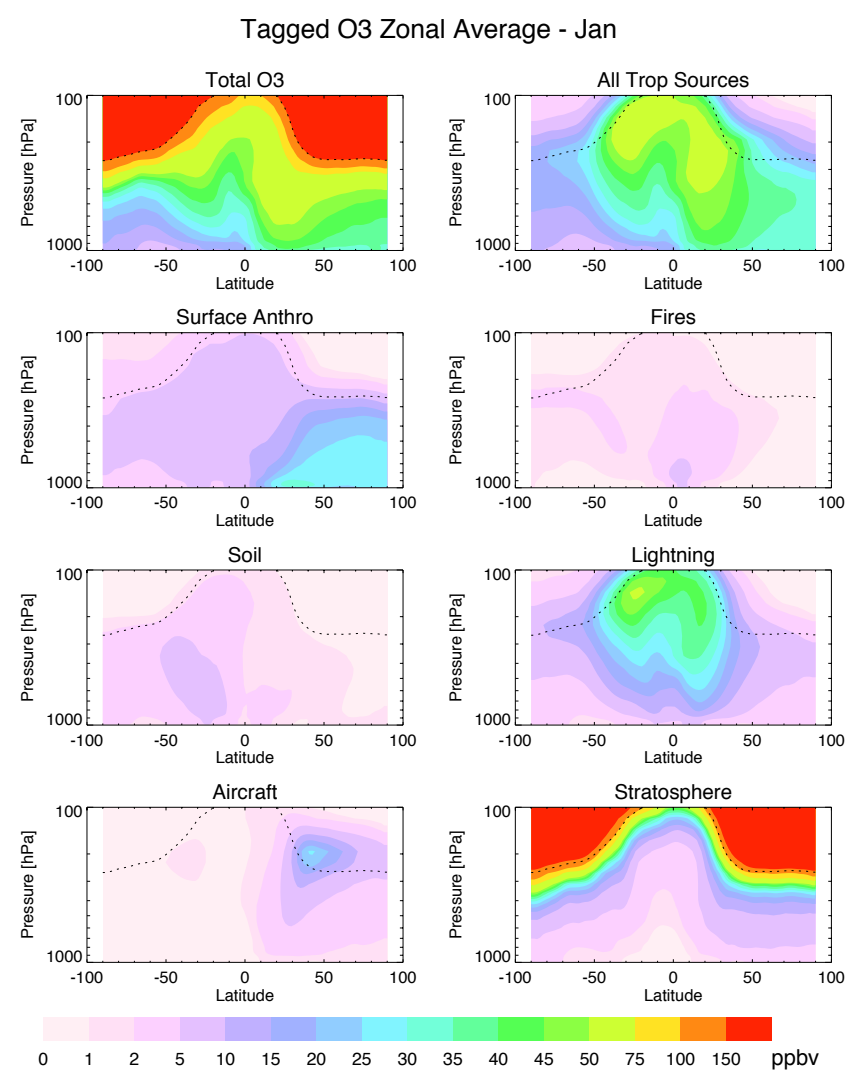

Fig. 4. Zonal average of each tagged ozone source for January 2008. Stratospheric contribution is determined as the difference between total ozone and tagged ozone from all tropospheric sources.

$\mathrm{HNO}_{3}$ shows the largest missing contribution between the sum of individual source tags and all sources (the white area in the plot between the lightning and stratosphere contributions). This is due to the nonlinearity of wet deposition and $\mathrm{HNO}_{3}$ is much more soluble than the other reactive nitrogen compounds. The washout rate for the tagged $\mathrm{HNO}_{3}$ is the same as for total $\mathrm{HNO}_{3}$, but the amount of tagged $\mathrm{HNO}_{3}$ will be limited by the available mass of that tag. So, at times (and places) all of the mass of a $\mathrm{HNO}_{3}$ tag may be removed from the atmosphere, eliminating any possibility of recycling to $\mathrm{NO}_{2}$ (through photolysis) and reformation of $\mathrm{HNO}_{3}$.

\subsection{Stratospheric ozone}

One commonly studied source of ozone is the stratospheric contribution to the troposphere. Many modelling studies have used a tracer ("O3S") that is set to the ozone mixing ratio in the stratosphere and is destroyed below the tropopause at the same rate as ozone (e.g., Roelofs and Lelieveld, 1997; Wang et al., 1998; Emmons et al., 2003). This technique, however, gives an upper limit to the stratospheric ozone contribution, as tropospheric air that is transported into the stratosphere is reset to the stratospheric value. By tagging all of the tropospheric sources (surface anthropogenic, biomass burning,
Tagged O3 Zonal Average - Jul
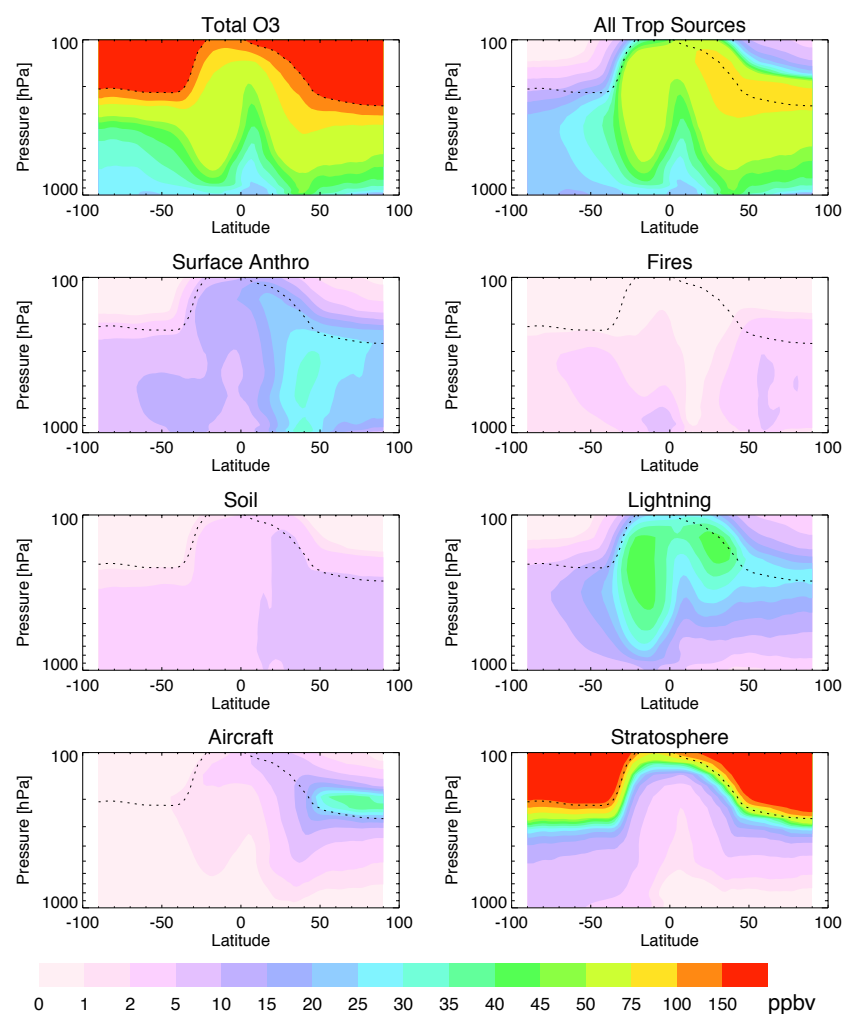

$\begin{array}{lllllllllllllllll}0 & 1 & 2 & 5 & 10 & 15 & 20 & 25 & 30 & 35 & 40 & 45 & 50 & 75 & 100 & 150 & \text { ppbv }\end{array}$

Fig. 5. As previous figure, for July 2008.

soil, lightning and aircraft emissions), we can determine the amount of ozone produced from tropospheric sources and, thus, the stratospheric contribution from the difference of the tagged amount and the total ozone.

Figure 8 shows the stratospheric contribution determined from the tagged tropospheric sources, as well as from the traditional "O3S" tracer. The O3S tracer gives a much greater estimate of stratospheric ozone than the tagged ozone does, both in the mid-troposphere and at the surface. At the surface, where O3S shows over $20 \mathrm{ppbv}$, the tagged ozone gives no more than 8 ppbv. Hess and Lamarque (2007) showed similar differences in zonal averages of surface O3S and tagged ozone. The vertical distributions of tagged ozone shown in Figs. 4 and 5 illustrate that a significant amount of tropospheric air gets into the stratosphere (i.e., above the $150 \mathrm{ppbv}$ ozone contour). With the O3S tracer, those contributions would get reset to stratospheric air, but the tagging method preserves those as tropospheric sources. When considering the whole troposphere (ozone less than $150 \mathrm{ppbv}$ ), the fraction of the tropospheric ozone burden that is from the stratosphere is $58 \%$ based on $\mathrm{O} 3 \mathrm{~S}$, and $17 \%$ based on the tagged ozone. These differences are comparable to those found using different measures by Emmons et al. (2003) in an analysis of the Northern Hemisphere springtime ozone increase. 

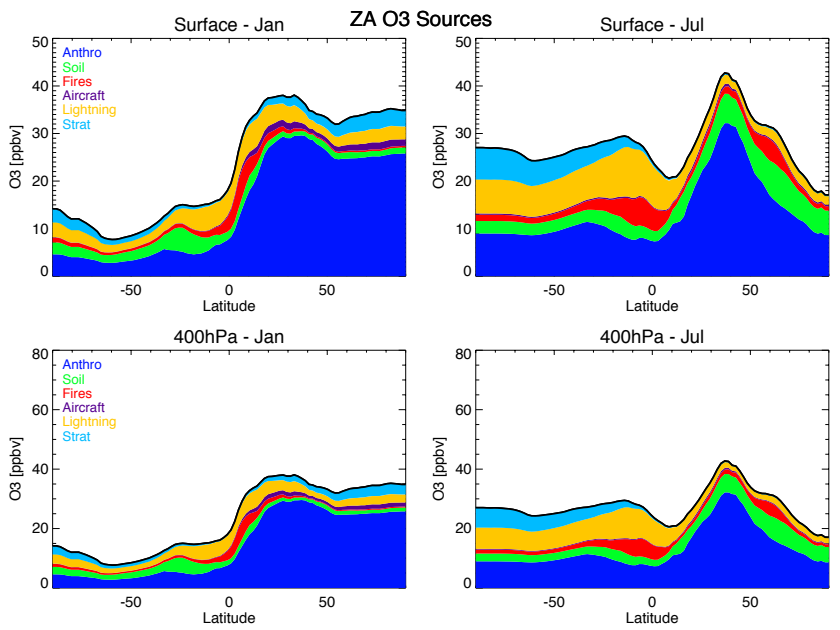

Fig. 6. Zonal average of tagged ozone source contributions at the surface and at $400 \mathrm{hPa}$, for January and July of 2008. Stratospheric contribution is determined as the difference between total ozone and tagged ozone from all tropospheric sources combined.

\subsection{Comparison to perturbed source attribution}

Simulations with perturbed emissions are primarily used to quantify the impacts of changes in emissions scenarios, but have also been used to approximate total source contributions. As mentioned above, many previous studies have determined ozone contributions from source regions by decreasing the NO emissions of that region and scaling the difference between the perturbed run and a standard run (e.g., Fiore et al., 2009; Reidmiller et al., 2009). The differences between attributing source contributions through perturbation or tagging procedures have been presented by Grewe et al. (2010, 2012), illustrating the nonlinearity of ozone chemistry and its effect on attributing ozone sources. We have performed a similar analysis of our tagging scheme in comparison to perturbation results and show here that the nonlinearity of ozone chemistry has a significant impact on the conclusions drawn from perturbation simulations.

We have performed two simulations in this manner with NO emissions in Asia reduced by 5 and $20 \%$, so as to compare it with our method of tagging NO. Figure 9 shows the original NO surface emissions (shipping, anthropogenic, fires and soil) for July 2008, also indicating the Asian region that has been either tagged or perturbed. This region is roughly the combination of the South Asia and East Asia regions used in the HTAP simulations (e.g., Fiore et al., 2009). Figure 10 shows a comparison of surface ozone attributed to emissions in Asia using these two techniques for several receptor regions (the N. America and Europe regions are similar to, but not exactly the same as, the HTAP regions). The two methods give notably different results. However, the two perturbation cases give essentially the same results when scaled to correspond to $100 \%$ attribution. Comparable
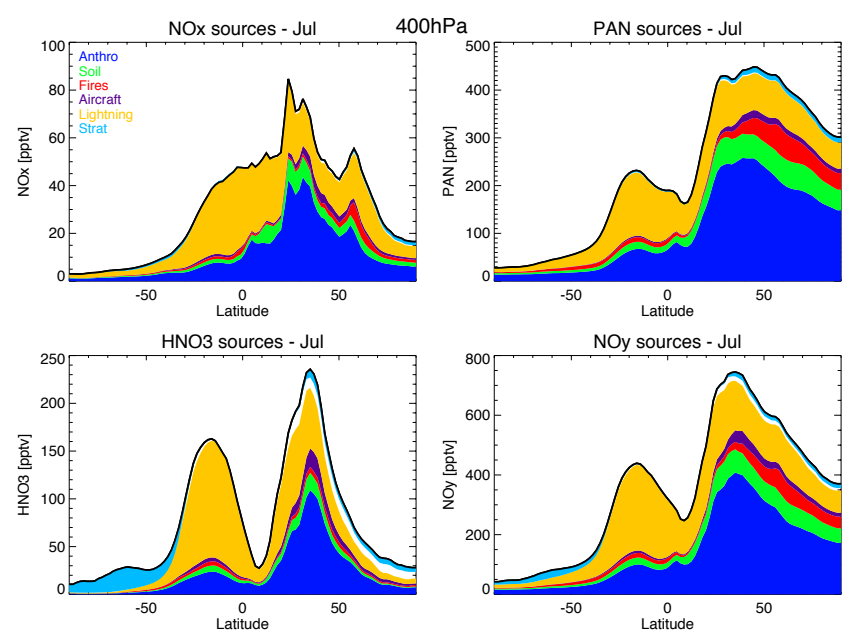

Fig. 7. As Fig. 6, for tagged $\mathrm{NO}_{\mathrm{x}}, \mathrm{PAN}, \mathrm{HNO}_{3}$ and $\mathrm{NO}_{\mathrm{y}}$ source contributions at $400 \mathrm{hPa}$ in July.

results were seen in other models with different chemical schemes (Wu et al., 2009; Wild et al., 2012). Over Asia, the average surface ozone produced from sources in Asia is 20$25 \mathrm{ppbv}$ in the Tagged method, but 1-6 ppbv in either Perturbed method. The seasonal cycle is also different, with the Tagged method peaking in April and having a minimum in August, while the Perturbed method shows a broad summer maximum. In the downwind regions, the seasonal cycle is more similar in the two methods, with the magnitude of the differences decreasing while progressing from the northeast Pacific to North America to Europe.

The results from the simulations with perturbed emissions agree well with the results of the HTAP simulations presented in Fig. 11 of Fiore et al. (2009). In the HTAP results, the estimated contribution of East Asia in North America is about $1.5 \mathrm{ppbv}$ in spring and less than $1 \mathrm{ppbv}$ in summer. This matches our results for the Perturbed case in the N. America panel of Fig. 10. Brown-Steiner and Hess (2011) found a similar difference between the HTAP results and their simulations with CAM-chem using the same tagged ozone procedure as presented here. That study found tagged ozone from Asia at the surface in North America to be 2-2.5 times that determined by the HTAP perturbed attribution procedure.

Since these two procedures for determining ozone source contributions give such different results, it is important to understand the causes, as well as which questions each procedure can address. If one wants to determine the effect of a change in emissions (due to policy controls or climate change), then the perturbed method is appropriate. However, the tagged ozone mechanism quantifies the contribution for a given source for a given state of the atmosphere, without any change in the chemical composition, and is useful in determining the ozone chemical budget.

Although a perturbation of 5 or $20 \%$ in the emissions may seem small, it can have a significant impact on the local 

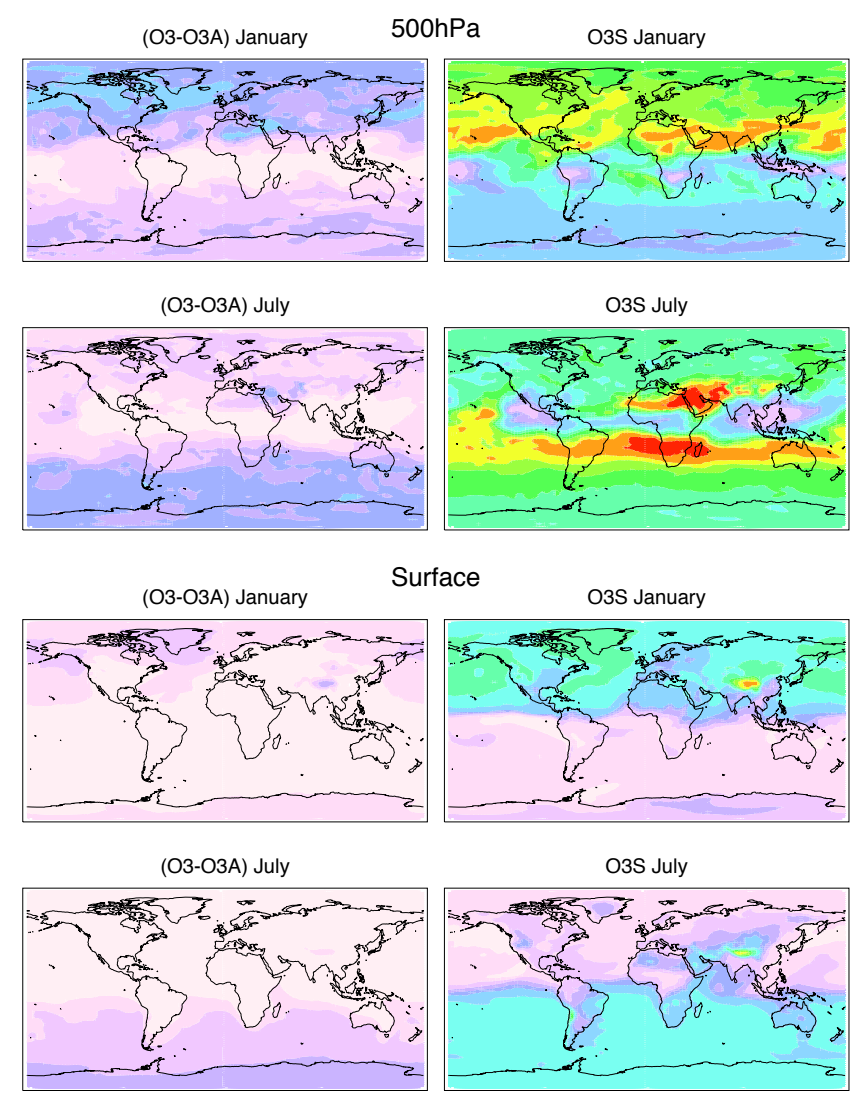

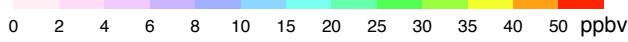

Fig. 8. Stratospheric ozone contribution from the tagged NO method (left) and from the stratospheric ozone tracer, O3S (right), at $500 \mathrm{hPa}$ (top) and the surface (bottom).

chemistry. In particular, the $\mathrm{OH}$ and $\mathrm{HO}_{2}$ distributions are very sensitive to changes in NO, which will lead to changes in the ozone production and loss rates. These effects were discussed in Pfister et al. (2006), in the analysis of ozone from biomass burning plumes using the tagged and perturbed methods, and are further illustrated here.

The rate-limiting reactions for ozone production and loss can be written as:

$P\left(\mathrm{O}_{3}\right)=[\mathrm{NO}] * K_{P}^{\prime}$ where $K_{P}^{\prime}=\left(k_{\mathrm{HO}_{2}}\left[\mathrm{HO}_{2}\right]+k_{\mathrm{RO} 2}\left[\mathrm{RO}_{2}\right]\right)$

$L\left(\mathrm{O}_{3}\right)=\left[\mathrm{O}_{3}\right] * K_{L}^{\prime}$ where $K_{L}^{\prime}=\left(k_{\mathrm{OH}}[\mathrm{OH}]+k_{\mathrm{HO}_{2}}\left[\mathrm{HO}_{2}\right]\right)$

For the tagged ozone, $P(\mathrm{O} 3 \mathrm{~A})=[\mathrm{XNO}] * K_{P}^{\prime}$ and $\mathrm{L}(\mathrm{O} 3 \mathrm{~A})=[\mathrm{O} 3 \mathrm{~A}] * K_{L}^{\prime}$ where the $K^{\prime}$ s are the same for the total ozone and tagged ozone rates because the $\mathrm{OH}$ and peroxy radicals are identical (because the tagged ozone is simultaneously calculated with the full chemistry). In the perturbed case, however, the change in $\mathrm{NO}$ emissions causes a change in $\mathrm{OH}, \mathrm{HO}_{2}, \mathrm{RO}_{2}$, etc., so the $K^{\prime}$ s are not the same between the base run and perturbed run. Figure 11 illustrates these differences in $K_{P}^{\prime}$ and $K_{L}^{\prime}$. Hourly output for the Base, Tagged

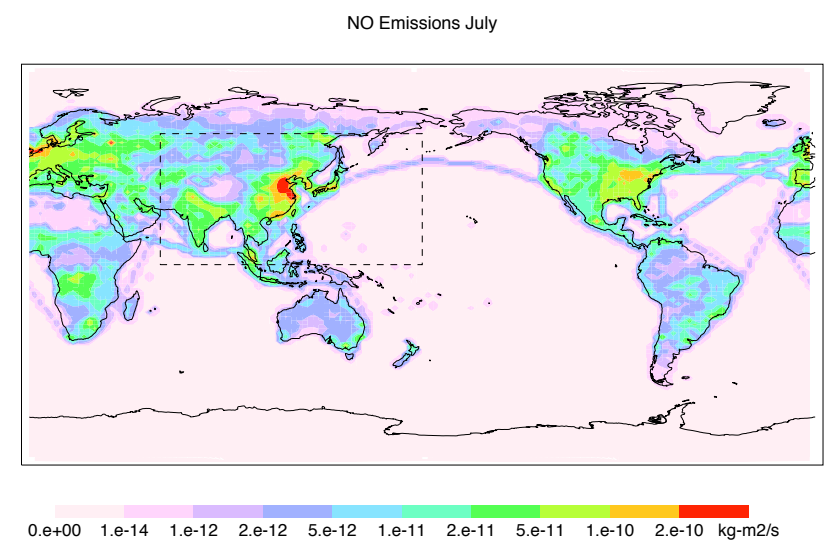

Fig. 9. NO surface emissions for July. Black dashed line shows region of tagged or perturbed emissions.
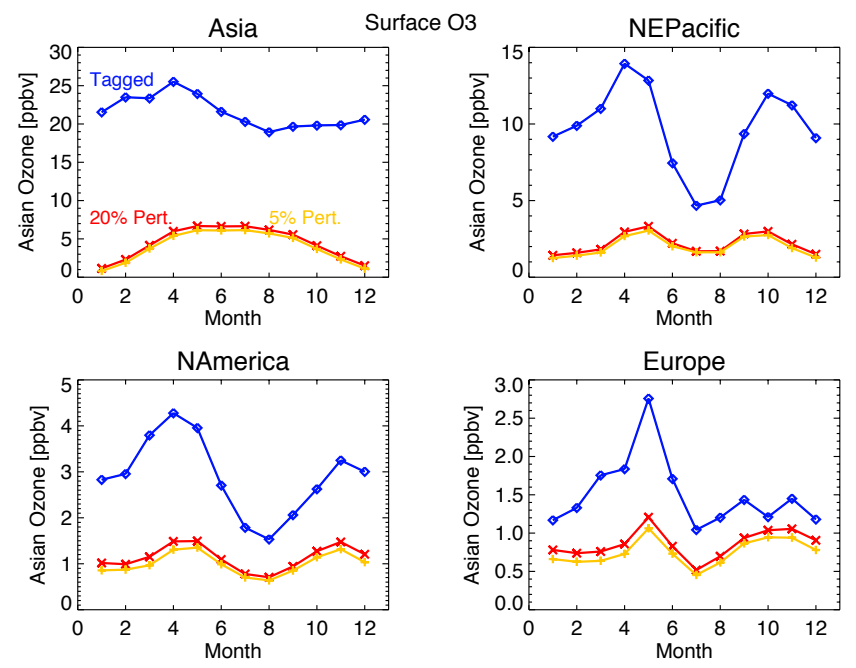

Fig. 10. Surface ozone attributed to Asian emissions based on tagged and perturbed $\mathrm{NO}_{\mathrm{x}}$ emissions, averaged over four receptor regions, as a function of month. The $20 \%$ perturbed contribution is 5 times (and the $5 \%$ perturbation case is 20 times) the difference between the standard simulation and a simulation with $80 \% \mathrm{NO}_{\mathrm{x}}$ emissions in Asia. The receptor regions are Asia $\left(0^{\circ}-60^{\circ} \mathrm{N}, 60^{\circ}-\right.$ $180^{\circ} \mathrm{E}$, same as emissions $)$, NE Pacific $\left(30^{\circ}-55^{\circ} \mathrm{N}, 190^{\circ}-235^{\circ} \mathrm{E}\right)$, N. America $\left(15^{\circ}-55^{\circ} \mathrm{N}, 235^{\circ}-300^{\circ} \mathrm{E}\right)$ and Europe $\left(30^{\circ}-70^{\circ} \mathrm{N}\right.$, $\left.0^{\circ}-45^{\circ} \mathrm{E}\right)$.

and Perturbed results are used to calculate $P\left(\mathrm{O}_{3}\right) /[\mathrm{NO}]$ and $L\left(\mathrm{O}_{3}\right) /\left[\mathrm{O}_{3}\right]$ to represent $K_{P}^{\prime}$ and $K_{L}^{\prime}$. The results shown are averages of the hourly calculations over 20-29 July, and then differenced with the Base calculation. For the tagged case, $K_{P}^{\prime}$ and $K_{L}^{\prime}$ are within a few percent of the Base case, whereas the perturbed $K_{P}^{\prime}$ is up to $15 \%$ higher than the Base case and $K_{L}^{\prime}$ is as much as $40 \%$ lower. These images also show that the nonlinearity effects vary significantly spatially, depending on the magnitude of $\mathrm{NO}_{\mathrm{x}}$ concentrations and the relative abundances of $\mathrm{NO}_{\mathrm{x}}$ and VOCs. Changes in $\mathrm{NO}_{\mathrm{x}}$ will 


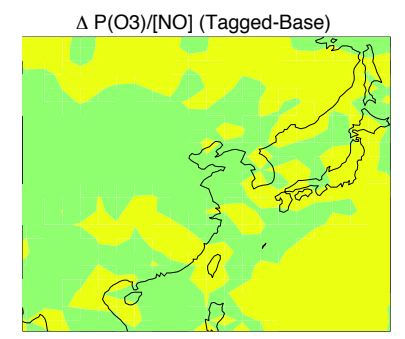

$\Delta \mathrm{L}(\mathrm{O} 3) /[\mathrm{O} 3]$ (Tagged-Base)
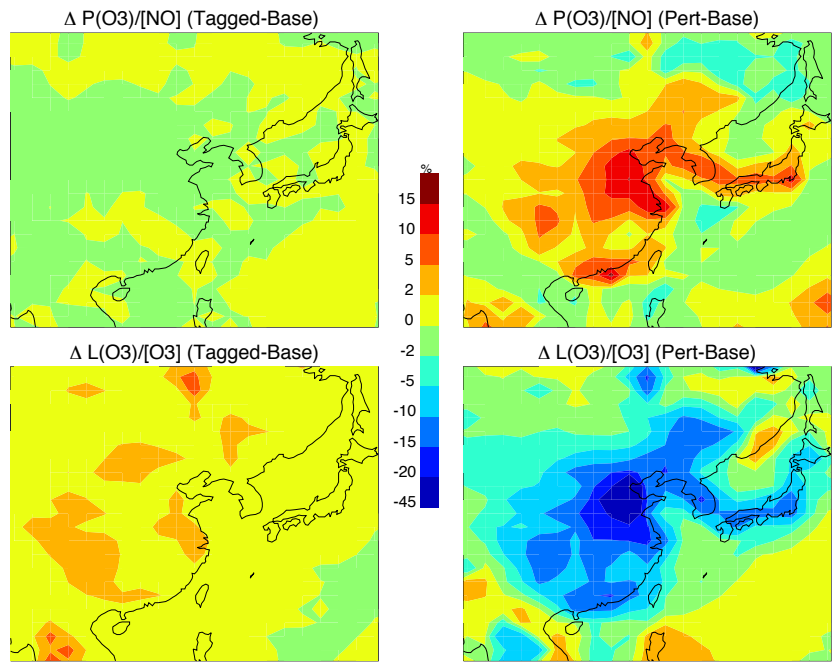

Fig. 11. Differences (\%) in $K_{P}^{\prime}$ and $K_{L}^{\prime}$ (see text) between Tagged and Base runs (left panels) and between Perturbed and Base runs (right). Average of hourly output for 20-29 July 2008.

affect ozone production and loss differently depending on the $\mathrm{NO}_{\mathrm{x}}$ and VOC levels. The greatest changes in $K_{P}^{\prime}$ and $K_{L}^{\prime}$ in the perturbed case are over the major urban areas of eastern Asia (Bejing, Shanghai, Pearl River Delta, Tokyo). Since these are the largest source regions they will significantly drive the regional averages shown in Fig. 10.

\section{Conclusions}

A technique of tagging ozone from various source regions by using artificial tracers of $\mathrm{NO}$ and its oxidation products has been described. This method shows a high degree of linearity: the sum of tagged ozone from individual sources is equal to the tagged ozone of all sources, within $3 \%$ in zonal averages in the troposphere. We have also shown, in a test simulation where the stratospheric input has been set to zero, that the tagged ozone from all tropospheric $\mathrm{NO}_{\mathrm{x}}$ sources is equal to the total ozone. This tagging procedure allows source contributions to $\mathrm{NO}_{\mathrm{x}}$ products, such as PAN and $\mathrm{HNO}_{3}$, to be quantified as well. Comparisons to other standard attribution methods show significant differences. The contribution of stratospheric ozone to the troposphere using this tagging method is significantly lower than estimates using a stratospheric ozone tracer. In comparison to determining source contributions by perturbing $\mathrm{NO}$ emissions, the tagging method gives significantly higher contributions, at least for an example for Asia emissions. These differences are due to the nonlinearity of ozone chemistry, where both ozone production and loss rates are affected by a change in NO concentrations.

The mechanism file and source code modifications for MOZART-4 and CAM-chem are available from the authors.
Acknowledgements. This article has been greatly improved as a result of the thoughtful reviews by M. Evans and T. Butler, helpful discussions with S. Arnold, and comments on earlier drafts by D. Kinnison and A. Boynard. The GEOS-5 data used in this study have been provided by the Global Modelling and Assimilation Office (GMAO) at NASA Goddard Space Flight Center. The National Center for Atmospheric Research is funded by the National Science Foundation.

Edited by: V. Grewe

\section{References}

Arellano, A. F., Kasibhatla, P. S., Giglio, L., van der Werf, G. R., Randerson, J. T., and Collatz, G. J.: Time-dependent inversion estimates of global biomass-burning $\mathrm{CO}$ emissions using Measurement of Pollution in the Troposphere (MOPITT) measurements, J. Geophys. Res., 111, D09303, doi:http://dx.doi.org/10.1029/2005JD00661310.1029/2005JD006613, 2006.

Brown-Steiner, B. and Hess, P.: Asian influence on surface ozone in the United tates: A comparison of chemistry, seasonality, and transport mechanisms, J. Geophys. Res., 116, D17309, doi:10.1029/2011JD015846, 2011.

Butler, T. M., Lawrence, M. G., Taraborrelli, D., and Lelieveld, J.: Multi-day ozone production potential of volatile organic compounds calculated with a tagging approach, Atmos. Environ., 45, 4082-4090, 2011.

Dahlmann, K., Grewe, V., Ponater, M., and Matthes, S.: Quantifying the contributions of individual $\mathrm{NO}_{\mathrm{x}}$ sources to the trend in ozone radiative forcing, Atmos. Environ., 45, 2860-2868, 2011.

Dentener, F., Keating, T., and Akimoto, H. (Eds.): Hemispheric Transport of Air Pollution, United Nations, available at: http: //htap.org (last access: January 2012), 2010.

Emmons, L., Hess, P., Klonecki, A., Tie, X., Horowitz, L., Lamarque, J. F., Kinnison, D., Brasseur, G., Atlas, E., Browell, E., Cantrell, C., Eisele, F., Mauldin, R. L., Merrill, J., Ridley, B., and Shetter, R.: Budget of tropospheric ozone during TOPSE from two chemical transport models, J. Geophys. Res., 108, 8372, doi:10.1029/2002JD002665, 2003.

Emmons, L. K., Apel, E. C., Lamarque, J.-F., Hess, P. G., Avery, M., Blake, D., Brune, W., Campos, T., Crawford, J., DeCarlo, P. F., Hall, S., Heikes, B., Holloway, J., Jimenez, J. L., Knapp, D. J., Kok, G., Mena-Carrasco, M., Olson, J., O'Sullivan, D., Sachse, G., Walega, J., Weibring, P., Weinheimer, A., and Wiedinmyer, C.: Impact of Mexico City emissions on regional air quality from MOZART-4 simulations, Atmos. Chem. Phys., 10, 6195-6212, doi:10.5194/acp-10-6195-2010, 2010a.

Emmons, L. K., Walters, S., Hess, P. G., Lamarque, J.-F., Pfister, G. G., Fillmore, D., Granier, C., Guenther, A., Kinnison, D., Laepple, T., Orlando, J., Tie, X., Tyndall, G., Wiedinmyer, C., Baughcum, S. L., and Kloster, S.: Description and evaluation of the Model for Ozone and Related chemical Tracers, version 4 (MOZART-4), Geosci. Model Dev., 3, 43-67, doi:10.5194/gmd3-43-2010, 2010b.

Fiore, A. M., Jacob, D. J., Bey, I., Yantosca, R. M., Field, B. D., Fusco, A. C., and Wilkinson, J. G.: Background ozone over 
the United States in summer: origin, trend, and contribution to pollution episodes, J. Geophys. Res., 107, 4275, doi:10.1029/2001JD000982, 2002.

Fiore, A. M., Dentener, F. J., Wild, O., Cuvelier, C., Schultz, M. G., Hess, P., Textor, C., Schulz, M., Doherty, R. M., Horowitz, L. W., MacKenzie, I. A., Sanderson, M. G., Shindell, D. T., Szopa, S., Van Dingenen, R., Zeng, G., Atherton, C., Bergmann, D., Bey, I., Stevenson, D. S., Carmichael, G., Collins, W. J., Duncan, B. N., Faluvegi, G., Folberth, G., Gauss, M., Gong, S., Hauglustaine, D., Holloway, T., Isaksen, I. S. A., Jacob, D. J., Jonson, J. E., Kaminski, J. W., Keating, T. J., Lupu, A., Marmer, E., Montanaro, V., Park, R. J., Pitari, G., Pringle, K. J., Pyle, J. A., Schroeder, S., Vivanco, M. G., Wind, P., Wojcik, G., Wu, S. and Zuber, A.: Multimodel estimates of intercontinental sourcereceptor relationships for ozone pollution, J. Geophys. Res., 114, D04301, doi:10.1029/2008JD010816, 2009.

Granier, C., Müller, J.-F., Pétron, G., and Brasseur, G.: A threedimensional study of the global CO budget, Chemosphere Glob. Change Sci., 1, 255-261, 1999.

Grewe, V.: Technical Note: A diagnostic for ozone contributions of various $\mathrm{NO}_{\mathrm{x}}$ emissions in multi-decadal chemistryclimate model simulations, Atmos. Chem. Phys., 4, 729-736, doi:10.5194/acp-4-729-2004, 2004.

Grewe, V., Tsati, E., and Hoor, P.: On the attribution of contributions of atmospheric trace gases to emissions in atmospheric model applications, Geosci. Model Dev., 3, 487-499, doi:10.5194/gmd3-487-2010, 2010.

Grewe, V., Dahlmann, K., Mathes, S., and Steinbrecht, W.: Attributing ozone to $\mathrm{NO}_{\mathrm{x}}$ emissions: Implications for climate mitigation measures, Atmos. Environ., 59, 102-107, 2012.

Guerova, G., Bey, I., Attié, J.-L., Martin, R. V., Cui, J., and Sprenger, M.: Impact of transatlantic transport episodes on summertime ozone in Europe, Atmos. Chem. Phys., 6, 2057-2072, doi:10.5194/acp-6-2057-2006, 2006.

Hauglustaine, D., Emmons, L., Newchurch, M., Brasseur, G., Takao, T., Matsubara, K., Johnson, J., Ridley, B., Stith, J., and Dye, J.: On the role of lightning $\mathrm{NO}_{\mathrm{x}}$ in the formation of tropospheric ozone plumes: a global model perspective, J. Atmos. Chem., 38, 277-294, doi:10.1023/A:1006452309388, 2001.

Hess, P. G. and Lamarque, J.-F.: Ozone source attribution and its modulation by the Arctic oscillation during the spring months, J. Geophys. Res., 112, D11303, doi:10.1029/2006JD007557, 2007.

Lamarque, J.-F., Brasseur, G. P., Hess, P. G., and Müller, J.-F.: Three-dimensional study of the relative contributions of the different nitrogen sources in the troposphere, J. Geophys. Res., 101, 955-968, 1996.

Lamarque, J.-F., Hess, P., Emmons, L., Buja, L., Washington, W., and Granier, C.: Tropospheric ozone evolution between 1890 and 1990, J. Geophys. Res., 110, D08304, doi:10.1029/2004JD005537, 2005.

Pétron, G., Granier, C., Khattotov, B., Yudin, V., Lamarque, J.F., Emmons, L., Gille, J., and Edwards, D. P.: Monthly CO surface sources inventory based on the 2000-2001 MOPITT satellite data, Geophys. Res. Lett., 31, L21107, doi:10.1029/2004GL020560, 2004.

Pfister, G., Pétron, G., Emmons, L. K., Gille, J. C., Edwards, D. P., Lamarque, J.-F., Attié, J.-L., Granier, C., and Novelli, P. C.: Evaluation of $\mathrm{CO}$ simulations and the analysis of the CO budget for Europe, J. Geophys. Res., 109, D19304,
doi:10.1029/2004JD004691, 2004.

Pfister, G. G., Emmons, L. K., Hess, P. G., Honrath, R., Lamarque, J.-F., Martin, M., Val and Owen, R. C., Avery, M. A., Browell, E. V., Holloway, J. S., Nedelec, P., Purvis, R., Ryerson, T. B., Sachse, G. W., and Schlager, H.: Ozone production from the 2004 North American boreal fires, J. Geophys. Res., 111, D24S07, doi:10.1029/2006JD007695, 2006.

Pfister, G. G., Emmons, L. K., Hess, P. G., Lamarque, J.-F., Orlando, J. J., Walters, S., Guenther, A., Palmer, P. I., and Lawrence, P. J.: Contribution of isoprene to chemical budgets: a model tracer study with the NCAR CTM MOZART-4, J. Geophys. Res., 113, D05308, doi:10.1029/2007JD008948, 2008a.

Pfister, G. G., Emmons, L. K., Hess, P. G., Lamarque, J.-F., Thompson, A. M., and Yorks, J. E.: Analysis of the Summer 2004 ozone budget over the United States using Intercontinental Transport Experiment Ozonesonde Network Study (IONS) observations and Model of Ozone and Related Tracers (MOZART-4) simulations, J. Geophys. Res., 113, D23306, doi:10.1029/2008JD010190, 2008b.

Pfister, G. G., Avise, J., Wiedinmyer, C., Edwards, D. P., Emmons, L. K., Diskin, G. D., Podolske, J., and Wisthaler, A.: CO source contribution analysis for California during ARCTASCARB, Atmos. Chem. Phys., 11, 7515-7532, doi:10.5194/acp11-7515-2011, 2011.

Price, C., Penner, J., and Prather, M.: $\mathrm{NO}_{\mathrm{x}}$ from lightning 1. Global distribution based on lightning physics, J. Geophys. Res., 102, 5929-5941, 1997.

Reidmiller, D. R., Fiore, A. M., Jaffe, D. A., Bergmann, D., Cuvelier, C., Dentener, F. J., Duncan, B. N., Folberth, G., Gauss, M., Gong, S., Hess, P., Jonson, J. E., Keating, T., Lupu, A., Marmer, E., Park, R., Schultz, M. G., Shindell, D. T., Szopa, S., Vivanco, M. G., Wild, O., and Zuber, A.: The influence of foreign vs. North American emissions on surface ozone in the US, Atmos. Chem. Phys., 9, 5027-5042, doi:10.5194/acp-9-5027-2009, 2009.

Roelofs, G.-J. and Lelieveld, J.: Model study of the influence of cross-tropopause $\mathrm{O}_{3}$ transports on tropospheric $\mathrm{O}_{3}$ levels, Tellus B, 49, 38-55, 1997.

Stevenson, D. S., Dentener, F. J., Schultz, M. G., Ellingsen, K., van Noije, T. P. C., Wild, O., Zeng, G., Amann, M., Atherton, C. S., Bell, N., Bergmann, D. J., Bey, I., Butler, T., Cofala, J., Collins, W. J., Derwent, R. G., Doherty, R. M., Drevet, J., Eskes, H. J., Fiore, A. M., Gauss, M., Hauglustaine, D. A., Horowitz, L. W., Isaksen, I. S. A., Krol, M. C., Lamarque, J. F., Lawrence, M. G., Montanaro, V., Muller, J. F., Pitari, G., Prather, M. J., Pyle, J. A., Rast, S., Rodriguez, J. M., Sanderson, M. G., Savage, N. H., Shindell, D. T., Strahan, S. E., Sudo, K., and Szopa, S.: Multimodel ensemble simulations of present-day and near-future tropospheric ozone, J. Geophys. Res., 111, D08301, doi:10.1029/2005JD006338, 2006.

Wang, Y., Jacob, D. J., and Logan, J. A.: Global simulation of tropospheric $\mathrm{O}_{3}-\mathrm{NO}_{\mathrm{x}}$-hydrocarbon chemistry, 3, Origin of tropospheric ozone and effects of nonmethane hydrocarbons, J. Geophys. Res., 103, 10757-10767, 1998.

Wespes, C., Emmons, L., Edwards, D. P., Hannigan, J., Hurtmans, D., Saunois, M., Coheur, P.-F., Clerbaux, C., Coffey, M. T., Batchelor, R. L., Lindenmaier, R., Strong, K., Weinheimer, A. J., Nowak, J. B., Ryerson, T. B., Crounse, J. D., and Wennberg, P. O.: Analysis of ozone and nitric acid in spring and 
summer Arctic pollution using aircraft, ground-based, satellite observations and MOZART-4 model: source attribution and partitioning, Atmos. Chem. Phys., 12, 237-259, doi:10.5194/acp12-237-2012, 2012.

Wiedinmyer, C., Akagi, S. K., Yokelson, R. J., Emmons, L. K., AlSaadi, J. A., Orlando, J. J., and Soja, A. J.: The Fire INventory from NCAR (FINN): a high resolution global model to estimate the emissions from open burning, Geosci. Model Dev., 4, 625641, doi:10.5194/gmd-4-625-2011, 2011.

Wild, O., Fiore, A. M., Shindell, D. T., Doherty, R. M., Collins, W. J., Dentener, F. J., Schultz, M. G., Gong, S., MacKenzie, I. A., Zeng, G., Hess, P., Duncan, B. N., Bergmann, D. J., Szopa, S., Jonson, J. E., Keating, T. J., and Zuber, A.: Modelling future changes in surface ozone: a parameterized approach, Atmos. Chem. Phys., 12, 2037-2054, doi:10.5194/acp-12-20372012, 2012.
Wu, S., Duncan, B. N., Jacob, D. J., Fiore, A. M., and Wild, O.: Chemical nonlinearities in relating intercontinental ozone pollution to anthropogenic emissions, Geophys. Res. Lett., 36, L05806, doi:10.1029/2008GL036607, 2009.

Zhang, Q., Streets, D. G., Carmichael, G. R., He, K. B., Huo, H., Kannari, A., Klimont, Z., Park, I. S., Reddy, S., Fu, J. S., Chen, D., Duan, L., Lei, Y., Wang, L. T., and Yao, Z. L.: Asian emissions in 2006 for the NASA INTEX-B mission, Atmos. Chem. Phys., 9, 5131-5153, doi:10.5194/acp-9-5131-2009, 2009. 